\title{
CONVERGENCE TO SUITABLE WEAK SOLUTIONS FOR A FINITE ELEMENT APPROXIMATION OF THE NAVIER-STOKES EQUATIONS WITH NUMERICAL SUBGRID SCALE MODELING
}

\author{
SANTIAGO BADIA ${ }^{\dagger}$ AND JUAN VICENTE GUTIÉRREZ-SANTACREU $\ddagger$
}

\begin{abstract}
In this work we prove that weak solutions constructed by a variational multiscale method are suitable in the sense of Scheffer. In order to prove this result, we consider a subgrid model that enforces orthogonality between subgrid and finite element components. Further, the subgrid component must be tracked in time. Since this type of schemes introduce pressure stabilization, we have proved the result for equal-order velocity and pressure finite element spaces that do not satisfy a discrete inf-sup condition.
\end{abstract}

2010 Mathematics Subject Classification: 35Q30; 65N30; 76N10.

Keywords: Navier-Stokes equations; Suitable weak solutions; Stabilized finite element methods, Subgrid scales.

\section{Contents}

1. Introduction

2. Statement of the problem 3

2.1. Notation 3

2.2. The Navier-Stokes equations 4

3. Finite element approximation 6

3.1. Hypotheses 6

3.2. The discrete problem 7

3.3. Discrete operators $\quad 8$

4. Technical preliminary results 9

5. A priori energy estimates 13

6. Convergence towards weak and suitable weak solutions 17

Appendix A. Proof of the inverse inequalities (8) 21

References 21

\section{INTRODUCTION}

Incompressible Newtonian fluids are governed by the Navier-Stokes equations. The existence of solutions is known from the works by Leray [30] and Hopf [26]. However, uniqueness is still an open question. The loss of regularity is related to turbulence [23], and Leray denoted weak solutions as

Date: December 22, 2016.

$\dagger$ Universitat Politècnica de Catalunya, Jordi Girona1-3, Edifici C1, E-08034 Barcelona \& Centre Internacional de Mètodes Numèrics en Enginyeria, Parc Mediterrani de la Tecnologia, Esteve Terrades 5, E-08860 Castelldefels, Spain Email: sbadia@cimne.upc.edu. SB was partially supported by by the European Research Council under the FP7 Program Ideas through the Starting Grant No. 258443 - COMFUS: Computational Methods for Fusion Technology and the FP7 NUMEXAS project under grant agreement 611636. SB gratefully acknowledges the support received from the Catalan Government through the ICREA Acadèmia Research Program.

$\ddagger$ Dpto. de Matemática Aplicada I, E. T. S. I. Informática, Universidad de Sevilla. Avda. Reina Mercedes, s/n. E41012 Sevilla, Spain. E-mail: juanvi@us.es. JVGS was partially supported by the Spanish grant No. MTM2015-69875-P from Ministerio de Economía y Competitividad with the participation of FEDER. 
turbulent solution. Scheffer defined the concept of suitable weak solutions in [34] and proved a bound for the Haussdorff dimension of the singular set of a weak suitable solution. This result was later improved by Cafarelli, Kohn, and Nirenberg [9], proving that this dimension is smaller than 1. This is the sharpest regularity result so far.

Suitable weak solutions of the Navier-Stokes equations can be constructed by regularization (see, e.g., [32]). More recently, Guermond proved that inf-sup stable finite element (FE) approximations having a discrete commutator property also converge to suitable weak solutions, first for periodic boundary conditions in the three-dimensional torus [21], and next on general domains and no-slip boundary conditions [22]. The Fourier method does not satisfy the required assumptions, and it is still an open question whether it provides suitable solutions.

The Navier-Stokes equations have a dissipative structure, due to the viscous term. The system has a singular limit in the assymptotic regime as the Reynolds (Re) number, which is the ratio of inertia forces to viscous forces, goes to infinity. The singular limit and the fact that the system is indefinite complicate its numerical approximation. The first property requires to introduce some kind of convection stabilization, whereas the second prevents the use of the same FE space for both the velocity and pressure unknowns, the discrete system is unstable.

At the continuous level, the nonlinear convective term transfers energy from the largest to the smallest scales, till reaching the Kolmogorov scale, where energy is dissipated. In direct numerical simulations (DNS) the mesh needs to be fine enough to capture the smallest scales in the flow. However, this approach is unacceptable for industrial turbulent flows, due to the limits in computational resources. In real applications, under-resolved simulations are needed. The smallest scales that can be captured in these simulations are far from the Kolmogoroy scale and dissipation is negligible. Thus,

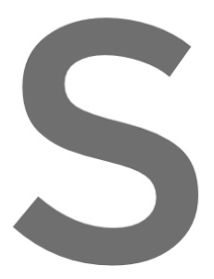
one has to add so-called
mechanisms. The concer
physically consistent way
grid scales, leading to in
Convection stabilizati
have considered so-called large
ht of su
stabilit
ion and
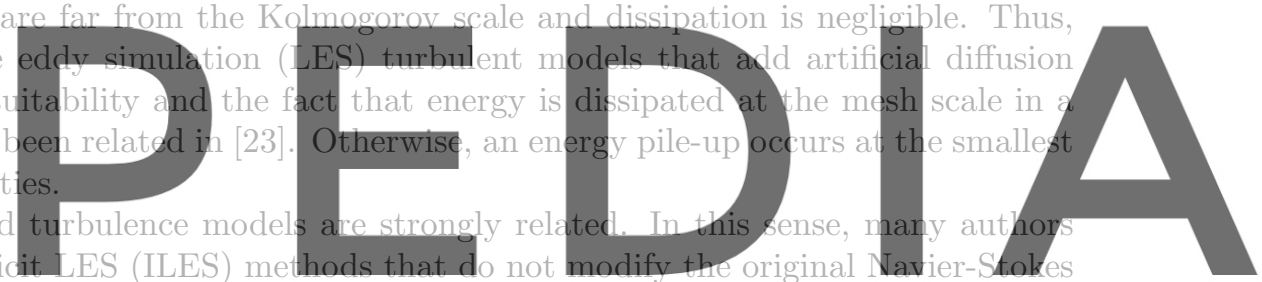

equations but introduce additional numerical artifacts when carrying out the discretization $[7,17]$.

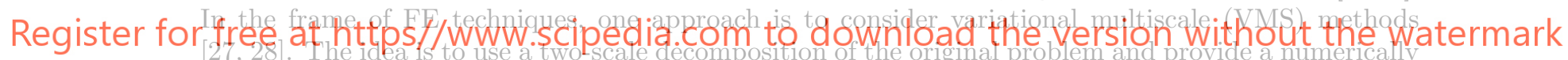
motivated closure for the fine scale (see, e.g., [20]). A similar stabilization procedure can be used for the convective term and the pressure term, leading to methods that do not require to satisfy a discrete inf-sup condition. An alternative to traditional residual-based methods is to consider subscales that are in some sense orthogonal to the FE space. This idea has been proposed by Codina [11], where $L^{2}(\Omega)$ orthogonality was used. This method involves global projections, which has motivated the use of local projections (see, e.g., [5, 2]). The treatment of the time dimension in the subgrid model has also been object of active research. In particular, the use of dynamic subscales methods that track the subgrid scale in time have been proposed in [11].

Even though DNS is impractical in real applications, it is better understood than stabilized or ILES schemes. The groundbreaking works by Guermond have proved that the FE Galerkin method leads to weak suitable solutions in [21, 22]. However, the extension to ILES methods is not straightforward, due to the introduction of additional terms to the numerical formulation. The analysis of these methods has usually been restricted to a priori error estimates for smooth enough solution (see, e.g., [10]). Residualbased VMS schemes are not amenable for weak convergence analysis, due to the proliferation of terms, e.g., including new velocity-pressure coupling terms. However, enforcing the modelled subgrid scales to be orthogonal to the FE space and considering the dynamic formulation in [11], the authors have proved in [4] that the resulting scheme converges to weak (turbulent) solutions of the Navier-Stokes equations. For the same scheme, long-term stability estimates and existence of a global attractor have been proved in [3]. Further, a very detailed numerical experimentation of these methods for isotropic and wall-bounded turbulent flows can be found in [12], proving that these subgrid models act as accurate turbulence models. Theoretical analyses supporting these results can also be found in [19]. 
In this work, we want to analyze whether VMS-type FE ILES schemes converge to suitable weak solutions in the sense of Scheffer. We prove that subgrid closures that are orthogonal and dynamic converge in fact to suitable solutions for equal order FE pairs for the velocity and pressure unknowns.

The outline of the work is the following. First, we state the problem and introduce the notation in Section 2. The FE approximation based on the VMS-type ILES scheme is introduced in Section 3. Section 4 includes some technical results in fractional Sobolev spaces. Energy estimates are proved in Section 5. Finally, the convergence towards weak and suitable solutions is proved in 6 .

\section{Statement of the Problem}

Throughout this paper we follow faithfully the notation used in [25] and [22] so that the reader can trace with ease the main differences between these two works and the one presented herein.

2.1. Notation. Let $\Omega$ be an open subset of $\mathbb{R}^{3}$. For $p \in[1, \infty]$, we denote by $L^{p}(\Omega)$ the usual Lebesgue space, i.e.,

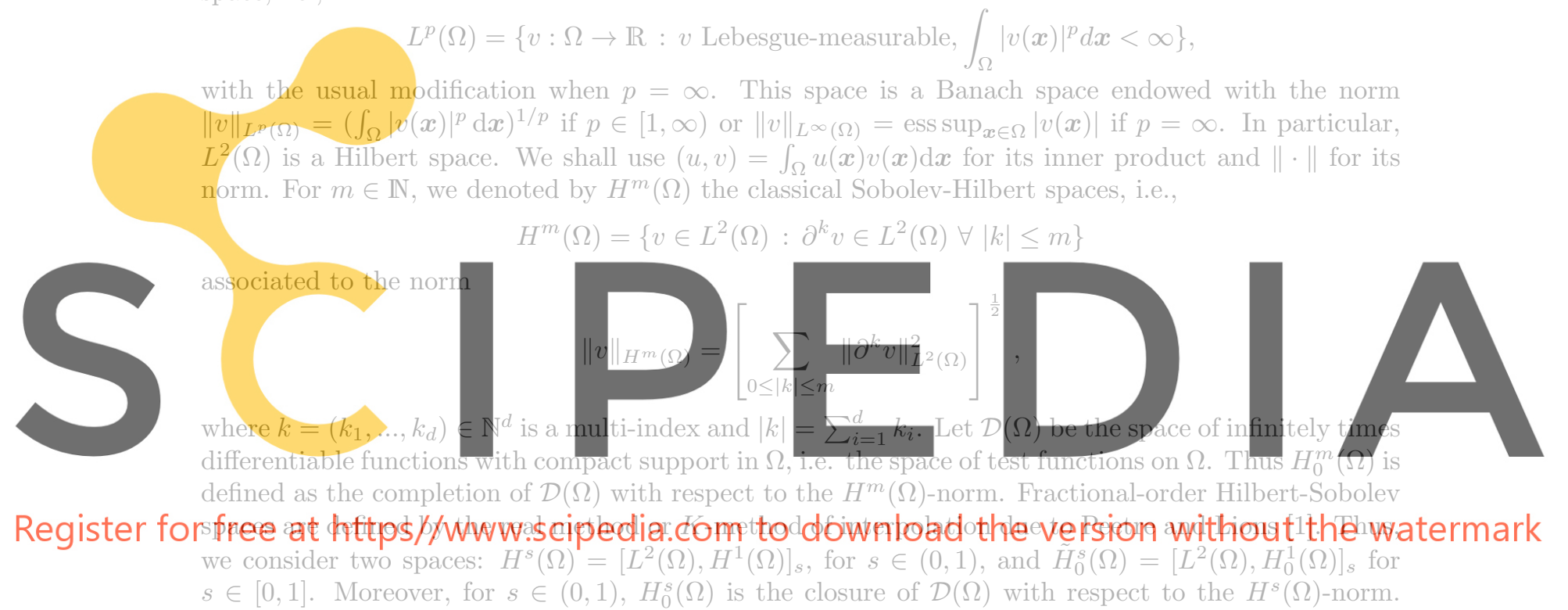

Note that the spaces $H^{s}(\Omega)$ and $H_{0}^{s}(\Omega)$ coincide for $s \in\left[0, \frac{1}{2}\right]$, with uniform norms [24, Th 11.1], and the spaces $H^{s}(\Omega)$ and $\tilde{H}_{0}^{s}(\Omega)$ coincide with equivalent norms [33] for $s \in\left[0, \frac{1}{2}\right)$. We also consider $H^{s}(\Omega)=\left[H^{1}(\Omega), H^{2}(\Omega)\right]_{s}$ for $s \in(1,2]$ and $\tilde{H}_{0}^{s}(\Omega)=H^{s}(\Omega) \cap H_{0}^{1}(\Omega)$ for $s \in(1,2]$.

The dual space of $\mathcal{D}(\Omega)$, the space of distributions, is denoted by $\mathcal{D}^{\prime}(\Omega)$. Moreover, for $s<0, \tilde{H}^{s}(\Omega)$ is the dual of $\tilde{H}_{0}^{-s}(\Omega)$ and the space $H_{0}^{-s}(\Omega)$ is the complexion of $\mathcal{D}(\Omega)$ under the norm

$$
\|v\|_{H^{-s}(\Omega)}=\sup _{w \in \mathcal{D}(\Omega) \backslash\{\mathbf{0}\}} \frac{(v, w)}{\|w\|_{H^{s}(\Omega)}},
$$

We use $\langle\cdot, \cdot\rangle$ to denote the duality pairing. For $s \in\left[0, \frac{1}{2}\right) \cup\left(\frac{1}{2}, \frac{3}{2}\right), H^{-s}(\Omega)$ coincides with $\tilde{H}_{0}^{-s}(\Omega)$.

We will use boldfaced letters for spaces of vector functions, e.g. $\boldsymbol{L}^{2}(\Omega)$ in place of $L^{2}(\Omega)^{d}$.

We will make use of the following space of vector fields:

$$
\boldsymbol{\vartheta}=\{\boldsymbol{v} \in \mathcal{D}(\Omega): \nabla \cdot \boldsymbol{v}=0 \text { in } \Omega\} .
$$

Related to the space $\boldsymbol{\vartheta}$, we consider the closures in the $\boldsymbol{L}^{2}(\Omega)$ and $\boldsymbol{H}^{1}(\Omega)$-norm, which are characterized by

$$
\begin{aligned}
\boldsymbol{H} & =\left\{\boldsymbol{u} \in \boldsymbol{L}^{2}(\Omega): \nabla \cdot \boldsymbol{u}=0 \text { in } \Omega, \boldsymbol{u} \cdot \boldsymbol{n}=0 \text { on } \partial \Omega\right\} \\
\boldsymbol{V} & =\left\{\boldsymbol{u} \in \boldsymbol{H}^{1}(\Omega): \nabla \cdot \boldsymbol{u}=0 \text { in } \Omega, \boldsymbol{u}=\mathbf{0} \text { on } \partial \Omega\right\}
\end{aligned}
$$

where $\boldsymbol{n}$ is the outward normal to $\Omega$ on $\partial \Omega$. This characterization is true for locally Lipschitz-continuous domains (see [36, Theorems 1.4 and 1.6] for a detailed proof). Furthermore, $L_{\int=0}^{2}(\Omega)\left(\right.$ resp. $H_{\int=0}^{1}(\Omega)$ ) 
is the space of zero-average $L^{2}(\Omega)$-functions (resp. zero-average $H^{1}(\Omega)$-functions ). Thus, by the real method of interpolation, $H_{\int=0}^{s}(\Omega)=\left[L_{\int=0}^{2}(\Omega), H_{\int=0}^{1}(\Omega)\right]$ for $s \in(0,1)$ (see $[24]$ ).

Let $X$ be a Banach space. Thus, $L^{p}(a, b ; X)$ denotes the space of Bochner-measurable, $X$-valued functions on the interval $(0, T)$ such that $\int_{0}^{T}\|f(s)\|_{X}^{p} \mathrm{~d} s<\infty$ if $1 \leq p<\infty$ or $\operatorname{ess}_{\sup } \in(0, T)\|f(s)\|_{X}<$ $\infty$ if $p=\infty$.

Moreover, $W^{1,1}(0, T ; X)$ is the space of functions $f \in L^{1}(0, T ; X)$ and $\frac{\mathrm{d}}{\mathrm{d} s} f \in L^{1}(0, T ; X)$ such that $\int_{0}^{T}\left(\|f(s)\|_{X}+\left\|\frac{\mathrm{d}}{\mathrm{d} s} f(s)\right\|_{X}\right) \mathrm{d} s<\infty$ and $W_{0}^{1,1}(0, T ; X)$ is the closure of $\mathcal{D}(0, T ; X)$ with respect to the $W^{1,1}(0, T ; X)$-norm, with $\mathcal{D}(0, T ; X)$ being the space of infinitely times differentiable functions defined on $(0, T)$ having values into $X$ with compact support in $(0, T)$. Additionally, the dual space of $W_{0}^{1,1}(0, T ; X)$ is denoted by $W^{-1, \infty}\left(0, T ; X^{\prime}\right)$ provided that $X$ is separable and reflexive.

The Fourier transform of a function $f \in L^{1}(\mathbb{R} ; X)$ is denoted by

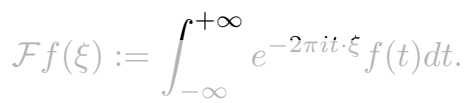

Let $H$ be a Hilbert space and let $\mathcal{S}^{\prime}(\mathbb{R} ; H)$ be the space of tempered distributions taking value in $H$. Thus, for $\gamma \in \mathbb{R}$, one defines

$$
H^{\gamma}(\mathbb{R} ; H)=\left\{v \in \mathcal{S}^{\prime}(\mathbb{R} ; H) ; \int_{\mathbb{R}}(1+|\xi|)^{2 \gamma}\|\mathcal{F} v\|_{H}^{2} d \xi\right\},
$$

where $H$ is a Hilbert space. Additionally, the space $H^{\gamma}(0, T ; H)$ is made up of tempered distributions
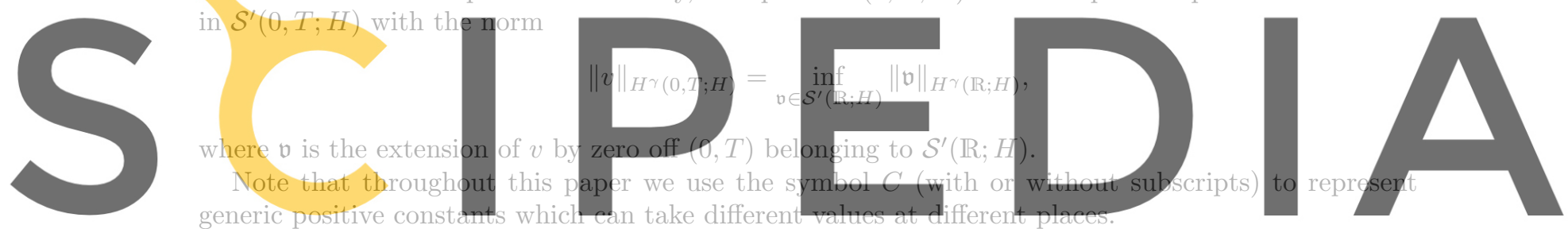

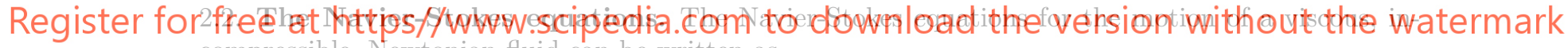
compressible, Newtonian fluid can be written as

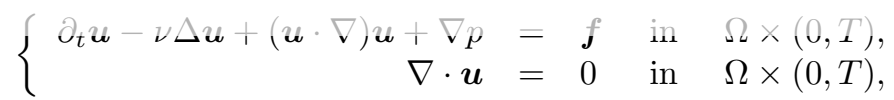

with $\Omega$ being a bounded, three-dimensional domain and with $0<T<+\infty$. Here $\boldsymbol{u}: \Omega \times(0, T) \rightarrow \mathbb{R}^{3}$ represents the incompressible fluid velocity and $p: \Omega \times(0, T) \rightarrow \mathbb{R}$ represents the fluid pressure. Moreover, $\boldsymbol{f}$ is the external body force which acts on the system, and $\nu>0$ is the kinematic fluid viscosity.

These equations are supplemented by the no-slip boundary condition

$$
\boldsymbol{u}=\mathbf{0} \quad \text { on } \quad \partial \Omega \times(0, T),
$$

and the initial condition

$$
\boldsymbol{u}(0)=\boldsymbol{u}_{0} \quad \text { in } \quad \Omega .
$$

The first authors dealing with the concept of weak solutions for the Navier-Stokes equations were Leray [30] for the Cauchy problem in the whole space and later Hopf [26] for the initial-boundary value problem in bounded domains. Particularly, weak solutions were called turbulent by Leray due to the possible connection between the lack of regularity of weak solutions and turbulence.

Definition 2.1. A function $\boldsymbol{u}$ is said to be a weak solution of problem (1)-(2) if:

$$
\boldsymbol{u} \in L^{\infty}(0, T ; \boldsymbol{H}) \cap L^{2}(0, T ; \boldsymbol{V})
$$


and

$$
\begin{aligned}
& -\int_{0}^{T}\left(\boldsymbol{u}(t), \partial_{t} \boldsymbol{v}(t)\right) \mathrm{d} t+\int_{0}^{T}\langle(\boldsymbol{u}(t) \cdot \nabla) \boldsymbol{u}(t), \boldsymbol{v}(t)\rangle \mathrm{d} t+\int_{0}^{T} \nu(\nabla \boldsymbol{u}(t), \nabla \boldsymbol{v}(t)) \mathrm{d} t \\
& =\left(\boldsymbol{u}_{0}, \boldsymbol{v}(0)\right)+\int_{0}^{T}\langle\boldsymbol{f}(t), \boldsymbol{v}(t)\rangle \mathrm{d} t
\end{aligned}
$$

for all $\boldsymbol{v} \in W^{1,1}(0, T ; \boldsymbol{V})$ with $\boldsymbol{v}(T)=\mathbf{0}$. Moreover, the energy inequality

$$
\frac{1}{2}\|\boldsymbol{u}(t)\|^{2}+\nu \int_{0}^{t}\|\nabla \boldsymbol{u}(s)\|^{2} \mathrm{~d} s \leq \frac{1}{2}\left\|\boldsymbol{u}_{0}\right\|^{2}+\int_{0}^{t}\langle\boldsymbol{f}(s), \boldsymbol{u}(s)\rangle \mathrm{d} s
$$

holds a. e. in $[0, T]$.

An equivalent definition for weak solutions involving the pressure term is defined as follows.

Definition 2.2. A pair $(\boldsymbol{u}, p)$ is said to be a weak solution of problem (1)-(2) if:

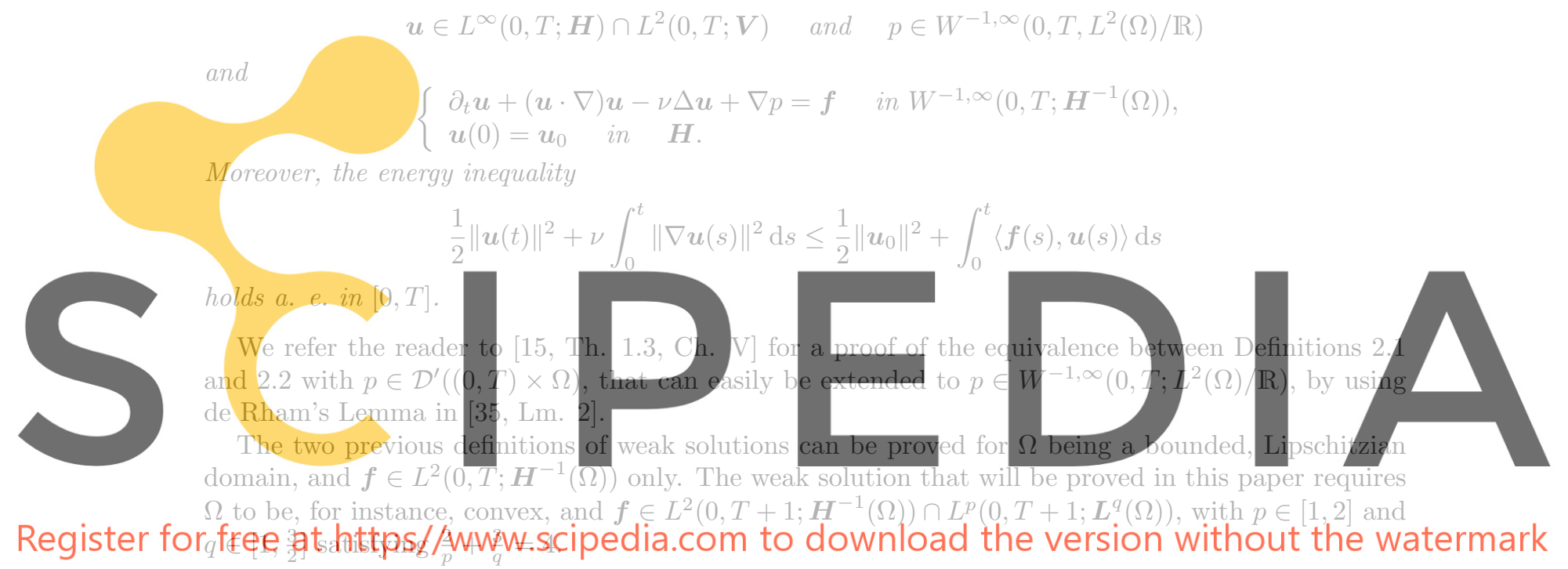

Definition 2.3. A pair $(u, p)$ is said to be a weak solution of problem (1)-(2) if:

$$
\boldsymbol{u} \in L^{\infty}(0, T ; \boldsymbol{H}) \cap L^{2}(0, T ; \boldsymbol{V}) \quad \text { and } \quad p \in H^{-r}\left(0, T, H_{\int=0}^{1-s}(\Omega)\right)
$$

with $s \in\left(\frac{1}{2}, \frac{7}{10}\right]$ and $r>\bar{r}=\frac{3}{4}-\frac{s}{2}$, and

$$
\left\{\begin{array}{rlllll}
\partial_{t} \boldsymbol{u}+(\boldsymbol{u} \cdot \nabla) \boldsymbol{u}-\nu \Delta \boldsymbol{u}+\nabla p & = & \boldsymbol{f} & \text { in } & H^{-r}\left(0, T ; \tilde{\boldsymbol{H}}_{0}^{-s}(\Omega)\right) \\
\boldsymbol{u}(0) & = & \boldsymbol{u}_{0} & \text { in } & \boldsymbol{H}
\end{array}\right.
$$

Moreover, the energy inequality

$$
\frac{1}{2}\|\boldsymbol{u}(t)\|^{2}+\nu \int_{0}^{t}\|\nabla \boldsymbol{u}(s)\|^{2} \mathrm{~d} s \leq \frac{1}{2}\left\|\boldsymbol{u}_{0}\right\|^{2}+\int_{0}^{t}\langle\boldsymbol{f}(s), \boldsymbol{u}(s)\rangle \mathrm{d} s
$$

holds a. e. in $[0, T]$.

Scheffer [34] introduced the definition of suitable weak solutions so as to prove a partial regularity theorem. Afterwards, Caffarelli, Kohn, and Nirenberg [9] improved Scheffer's results, and F.-H. Lin [31] simplified the proofs of the results in [9].

Definition 2.4. A weak solution $(\boldsymbol{u}, p)$ is said to be suitable if the local energy inequality

$$
\partial_{t}\left(\frac{1}{2} \boldsymbol{u}^{2}\right)+\nabla \cdot\left(\left(\frac{1}{2} \boldsymbol{u}^{2}+p\right) \boldsymbol{u}\right)-\nu \Delta\left(\frac{1}{2} \boldsymbol{u}^{2}\right)+\nu(\nabla \boldsymbol{u})^{2}-\boldsymbol{f} \cdot \boldsymbol{u} \leq 0
$$

holds in $\mathcal{D}^{\prime}\left((0, T) \times \Omega ; \mathbb{R}^{+}\right)$. 


\section{Finite element approximation}

3.1. Hypotheses. Throughout this paper we will assume the following hypotheses:

(H1) Let $\Omega$ be a connected, bounded, open subset of $\mathbb{R}^{3}$ having a polyhedral boundary such that there exist $\boldsymbol{v} \in \boldsymbol{V} \cap \boldsymbol{H}^{2}(\Omega)$ and $p \in H_{\int=0}^{1}(\Omega)$ satisfying

$$
\begin{array}{rlll}
-\Delta \boldsymbol{v}+\nabla p & =\boldsymbol{g} & \text { in } & \Omega, \\
\nabla \cdot \boldsymbol{v} & =0 & \text { in } & \Omega .
\end{array}
$$

(H2) Consider $\left\{\mathcal{T}_{h}\right\}_{h>0}$ to be a shape-regular and quasi-uniform family of simplicial and conforming meshes of $\Omega$ such that $\bar{\Omega}=\cup_{K \in \mathcal{T}_{h}} K$ with $h=\max _{K \in \mathcal{T}_{h}} h_{K}$ where $h_{K}=\operatorname{diam} K$.

(H3) Let $\left\{\boldsymbol{W}_{h}\right\}_{h>0}$ and $\left\{Q_{h}\right\}_{h>0}$ be two families of finite-element spaces associated with $\left\{\mathcal{T}_{h}\right\}_{h>0}$ such that $\boldsymbol{W}_{h} \subset \boldsymbol{H}_{0}^{1}(\Omega)$ and $Q_{h} \subset H_{\int=0}^{1}(\Omega)$. Moreover, the finite-element spaces are required to satisfy the following conditions. Let $\pi_{\boldsymbol{W}_{h}}: \boldsymbol{L}^{2}(\Omega) \rightarrow \boldsymbol{W}_{h}$ and $\pi_{Q_{h}}: \boldsymbol{L}^{2}(\Omega) \rightarrow Q_{h}$ be the orthogonal projections onto $W_{h}$ and $Q_{h}$, respectively, with respect to the $L^{2}(\Omega)$-inner product. Furthermore, we denote $\pi_{W_{h}}^{\perp}(\cdot):=(\cdot)-\pi_{W_{h}}(\cdot)$ and $\pi_{Q_{h}}^{\perp}(\cdot):=(\cdot)-\pi_{Q_{h}}(\cdot)$. (a) There exists a constant $C_{\text {inv }}>0$, independent of $h$, such that, for all $w_{h} \in W_{h}$,

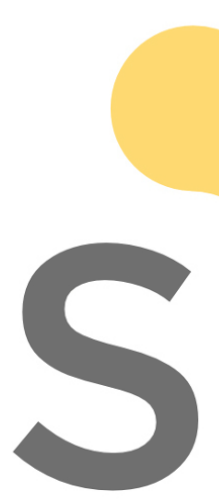

$$
\left\|\boldsymbol{w}_{h}\right\|_{\boldsymbol{L}^{\infty}(\Omega)} \leq C_{\mathrm{inv}} h^{-\frac{3}{k}}\left\|\boldsymbol{w}_{h}\right\|_{\boldsymbol{L}^{k}(\Omega)}
$$

\section{and}

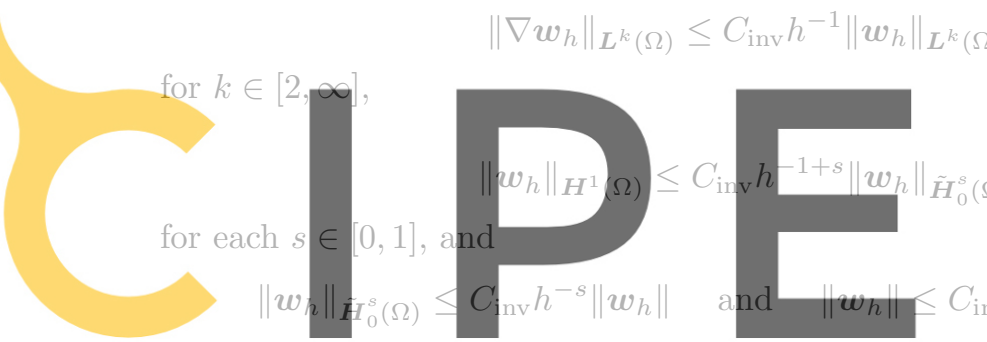

for $s \in[0,1]$.

$$
\left\|\nabla \boldsymbol{w}_{h}\right\|_{\boldsymbol{L}^{k}(\Omega)} \leq C_{\mathrm{inv}} h^{-1}\left\|\boldsymbol{w}_{h}\right\|_{\boldsymbol{L}^{k}(\Omega)}
$$

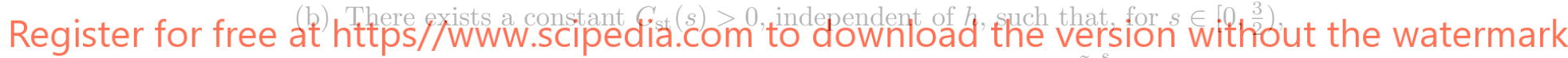
$\left\|\pi_{W_{h}} w\right\|_{\tilde{H}_{0}^{s}(\Omega)} \leq C_{\text {st }}(s)\|w\|_{\tilde{H}_{0}^{s}(\Omega)} \quad$ for all $\quad w \in \tilde{H}_{0}^{s}(\Omega)$,

(c) There exists a constant $C_{\text {int }}>0$, independent of $h$, such that, for all $l$ and $s$, satisfying $0 \leq l \leq \min \{1, s\}$ and $l \leq s \leq 2$, there holds

$$
\left\|\pi_{\boldsymbol{W}_{h}}^{\perp} \boldsymbol{w}\right\|_{\tilde{\boldsymbol{H}}_{0}^{l}(\Omega)} \leq C_{\mathrm{int}} h^{s-l}\|\boldsymbol{w}\|_{\tilde{\boldsymbol{H}}_{0}^{s}(\Omega)} \quad \text { for all } \quad \boldsymbol{w} \in \tilde{\boldsymbol{H}}_{0}^{s}(\Omega),
$$

and

$$
\left\|\pi_{Q_{h}}^{\perp} q\right\|_{H^{l}(\Omega)} \leq C_{\mathrm{int}} h^{s-l}\|q\|_{H^{s}(\Omega)} \quad \text { for all } \quad q \in H_{\int=0}^{s}(\Omega) .
$$

(d) There exists $C_{\text {com }}>0$, independent of $h$, such that, for $0 \leq l \leq m \leq 1$ and $\varphi \in W_{0}^{2, \infty}(\Omega)$,

$\left\|\pi_{\boldsymbol{W}_{h}}^{\perp}\left(\varphi \boldsymbol{w}_{h}\right)\right\|_{\boldsymbol{H}^{l}(\Omega)} \leq C h^{1+m-l}\left\|\boldsymbol{w}_{h}\right\|_{\boldsymbol{H}^{m}(\Omega)}\|\varphi\|_{W_{0}^{m+1, \infty}(\Omega)} \quad$ for all $\quad \boldsymbol{w}_{h} \in \boldsymbol{W}_{h}$,

and

$$
\left\|\pi_{Q_{h}}^{\perp}\left(\varphi q_{h}\right)\right\|_{\boldsymbol{H}^{l}(\Omega)} \leq C h^{1+m-l}\left\|q_{h}\right\|_{\boldsymbol{H}^{m}(\Omega)}\|\varphi\|_{W_{0}^{m+1, \infty}(\Omega)} \quad \text { for all } \quad q_{h} \in Q_{h} .
$$

(H4) Let $\boldsymbol{u}_{0} \in \boldsymbol{V}$ and $\boldsymbol{f} \in L^{2}\left(0, T+1 ; \boldsymbol{H}^{-1}(\Omega)\right) \cap L^{p}\left(0, T+1 ; \boldsymbol{L}^{q}(\Omega)\right)$, with $p \in[1,2]$ and $q \in\left[1, \frac{3}{2}\right]$ satisfying $\frac{2}{p}+\frac{3}{q}=4$.

Hypothesis (H1) is ensured for domains having a $C^{1,1}$ boundary or being a convex polygon (cf. [29] or [18]) or polyhedron (cf. [13]), with continuous dependence on $\boldsymbol{f}$.

Hypothesis (H3) is extremely flexible and allows equal-order finite-element spaces for velocity and pressure. For instance, let $\mathcal{P}_{k}(K)$ be the set of piecewise polynomial functions of degree less than or 
equal to $k$ on $K$ being a tetrahedra. Thus the space of continuous, piecewise polynomial functions of degree less than or equal to $k$ on a mesh $\mathcal{T}_{h}$ is denoted as

$$
X_{h}=\left\{v_{h} \in C^{0}(\bar{\Omega}):\left.v_{h}\right|_{K} \in \mathcal{P}_{k}(K), \forall K \in \mathcal{T}_{h}\right\},
$$

We choose the following continuous finite-element spaces

$$
\boldsymbol{W}_{h}=\boldsymbol{X}_{h} \cap \boldsymbol{H}_{0}^{1}(\Omega) \text { and } \quad Q_{h}=X_{h} \cap L_{\int=0}^{2}(\Omega),
$$

for approximating velocity and pressure, respectively.

The shape-regular and quasi-uniform properties of $\left\{\mathcal{T}_{h}\right\}_{h>0}$ assumed in (H2) suffice to ensure the properties of (H3)(a). We recommend the books [8, Sec. 4.5] and [14, Sec. 1.7] for a proof of (6) and (7), Appendix A for a proof of (8), and [16] for a proof of (9). Moreover, the error estimates stated in (H3) make use of (H2) as well (see [25, Lm A.3, Rm 2.1] for a proof).

The local approximation properties for the orthogonal projection operators $\pi_{W_{h}}$ and $\pi_{Q_{h}}$ guarantee hypothesis (II4). The reader is referred to [6].

Remark 3.1. Let $p$ and $q$ be as in (H4). We know from Sobolev's embeddings that $\tilde{\boldsymbol{H}}_{0}^{s}(\Omega)$ is embedded in $\boldsymbol{L}^{q^{\prime}}(\Omega)$, where $\frac{1}{q^{\prime}}+\frac{1}{q}=1$ and $s=3\left(\frac{1}{q}-\frac{1}{2}\right)$; hence $\boldsymbol{L}^{q}(\Omega)$ is embedded in $\tilde{\boldsymbol{H}}_{0}^{-s}(\Omega)$. Moreover, $H^{r}(\mathbb{R} ; H)$ is embedded in $L^{p^{\prime}}(\mathbb{R} ; H)$, where $\frac{1}{p^{\prime}}+\frac{1}{p}=1$ and $r>\bar{r}=\frac{1}{p}-\frac{1}{2}$ with $H$ being a Hilbert space; hence $L^{p}(\mathbb{R} ; H)$ is embedded in $H^{-r}(\mathbb{R} ; H)$. Let $\mathfrak{f}$ be the extension of $\boldsymbol{f}$ outside $[0, T]$ as zero. Then, by Hausdorff-Young's inequality for the Fourier transform, we have

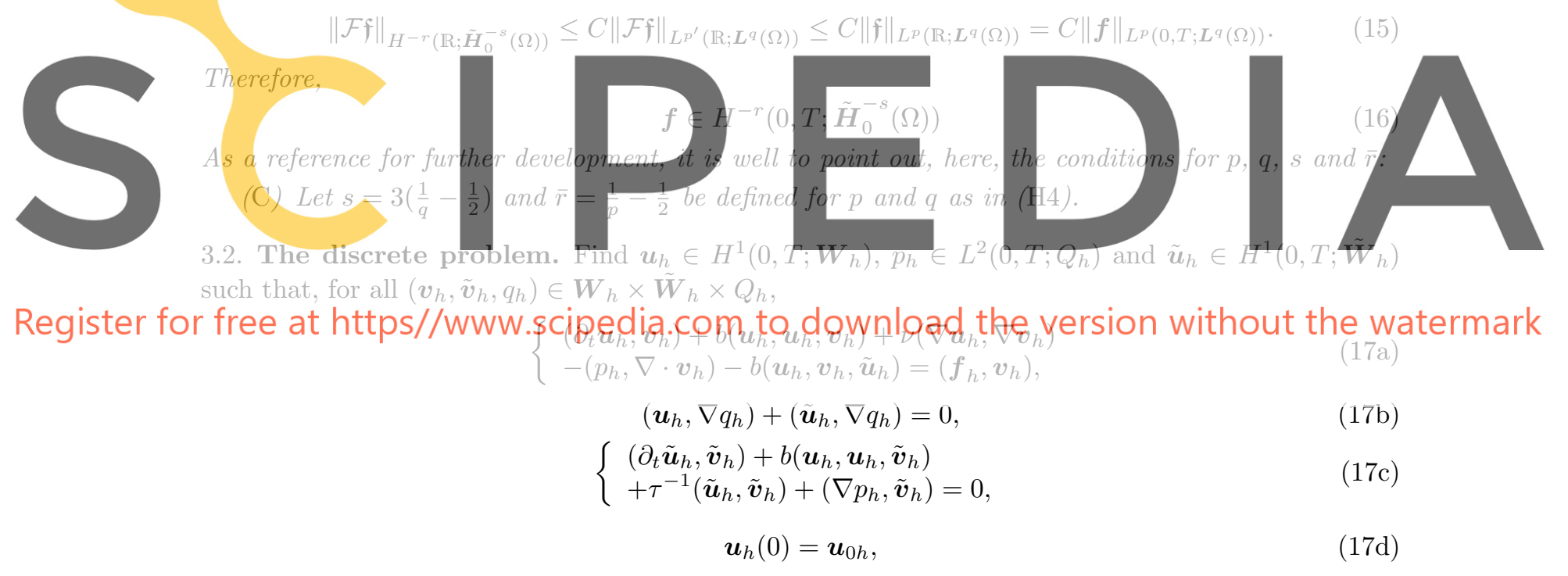

where

$$
\tau=\frac{1}{\frac{C_{s} \nu}{h^{2}}+\frac{C_{c}\left\|\boldsymbol{u}_{h}\right\|_{L^{\infty}(\Omega)}}{h}}=\frac{h^{2}}{C_{s} \nu+C_{c} h\left\|\boldsymbol{u}_{h}\right\|_{L^{\infty}(\Omega)}},
$$

with $C_{s}$ and $C_{c}$ being algorithmic positive constants, and $\boldsymbol{f}_{h} \in \boldsymbol{W}_{h}$ is defined by duality as $\left(\boldsymbol{f}_{h}, \boldsymbol{w}_{h}\right)=$ $\left\langle\boldsymbol{f}, \boldsymbol{w}_{h}\right\rangle$, for all $\boldsymbol{w}_{h} \in \boldsymbol{W}_{h}$. Let us define

$$
b\left(\boldsymbol{u}_{h}, \boldsymbol{v}_{h}, \boldsymbol{w}_{h}\right)=\left\langle\mathcal{N}\left(\boldsymbol{u}_{h}, \boldsymbol{v}_{h}\right), \boldsymbol{w}_{h}\right\rangle,
$$

where $\mathcal{N}\left(\boldsymbol{u}_{h}, \boldsymbol{v}_{h}\right)=\left(\boldsymbol{u}_{h} \cdot \nabla\right) \boldsymbol{v}_{h}+\frac{1}{2}\left(\nabla \cdot \boldsymbol{u}_{h}\right) \boldsymbol{v}_{h}$.

Let $\left\{\boldsymbol{\psi}_{i}\right\}_{i=1, \ldots, \mathrm{n}_{\mathrm{u}}}$ be a basis of $\boldsymbol{W}_{h}$ and let $\left\{\psi_{i}\right\}_{i=1, \ldots, \mathrm{n}_{p}}$ be a basis of $Q_{h}$, where $\mathrm{n}_{\mathrm{u}}$ and $\mathrm{n}_{p}$ denote the space dimension for $\boldsymbol{W}_{h}$ and $Q_{h}$, respectively. Thus, one defines

$$
\tilde{\boldsymbol{W}}_{h}=\operatorname{span}\left\{\pi_{\boldsymbol{W}_{h}}^{\perp}\left(\mathcal{N}\left(\phi_{i}, \phi_{j}\right)\right), \pi_{\boldsymbol{W}_{h}}^{\perp}\left(\nabla \phi_{k}\right)\right\},
$$

and $\boldsymbol{W}_{\star}=\boldsymbol{W}_{h} \oplus \tilde{\boldsymbol{W}}_{h}$. Moreover, one defines

$$
\boldsymbol{V}_{\star}=\left\{\boldsymbol{v}_{\star} \in \boldsymbol{W}_{\star}:\left(\boldsymbol{v}_{h}, \nabla q_{h}\right)+\left(\tilde{\boldsymbol{v}}_{h}, \nabla q_{h}\right)=0 \text { for all } q_{h} \in Q_{h}\right\} .
$$


which is a non-conforming approximation space of $\boldsymbol{V}$.

The initialization of the discrete problem can be obtained by the following projection problem: find $\boldsymbol{u}_{0 h} \in \boldsymbol{V}_{h}, \tilde{\boldsymbol{u}}_{0 h} \in \tilde{\boldsymbol{V}}_{h}$ and $\xi_{h} \in Q_{h}$ such that

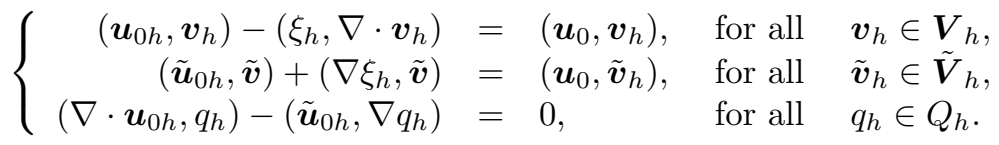

3.3. Discrete operators. This subsection is devoted to introducing the discrete operators that are used throughtout this paper.

Firstly, we will consider a conforming and non-conforming approximation of the Laplace operator $-\Delta: \tilde{\boldsymbol{H}}_{0}^{2}(\Omega) \rightarrow \boldsymbol{L}^{2}(\Omega)$. The non-conforming approximation is based on a stabilizing technique.

Consider $-\Delta_{h}: \boldsymbol{H}_{0}^{1}(\Omega) \rightarrow \boldsymbol{W}_{h}$ to be the discrete Laplacian operator defined as:

$$
-\left(\Delta_{h} \boldsymbol{w}, \overline{\boldsymbol{w}}_{h}\right)=\left(\nabla \boldsymbol{w}_{h}, \nabla \overline{\boldsymbol{w}}_{h}\right) \quad \text { for all } \quad \overline{\boldsymbol{w}}_{h} \in \boldsymbol{W}{ }_{h} .
$$

The restriction of this operator $-\Delta_{h}$ to $W_{h} \subset H_{0}^{1}(\Omega)$ gives a self-adjoint, positive-definite operator. Therefore, we are allowed to define the fractional power of $-\Delta_{h}$, say $\left(-\Delta_{h}\right)^{s}$, for all $s \in \mathbb{R}$, by the Hilbert-Schmidt theorem. The domain of definition of $\left(-\Delta_{h}\right)^{s}$ is $D\left(\left(-\Delta_{h}\right)^{s}\right) \equiv W_{h}$ since $\operatorname{dim} \boldsymbol{W}_{h}<$ $\infty$. Hence, $\boldsymbol{W}_{h}^{s}$ makes reference to $\boldsymbol{W}_{h}$ equipped with the Hilbert norm

$$
\left\|\boldsymbol{w}_{h}\right\|_{W_{h}^{s}}=\left(\left(-\Delta_{h}\right)^{\frac{s}{2}} \boldsymbol{w}_{h},\left(-\Delta_{h}\right)^{\frac{s}{2}} \boldsymbol{w}_{h}\right)^{\frac{1}{2}} .
$$

The family $\left\{\boldsymbol{W}_{h}^{s}\right\}_{s \in \mathbb{R}}$ is a scale of Hilbert spaces with respect to the real method of interpolation.

Analogously, consider $-_{\Delta_{\star}}: W_{\star} \rightarrow W_{\star}$ to be the stabilized discrete Laplacian operator defined as
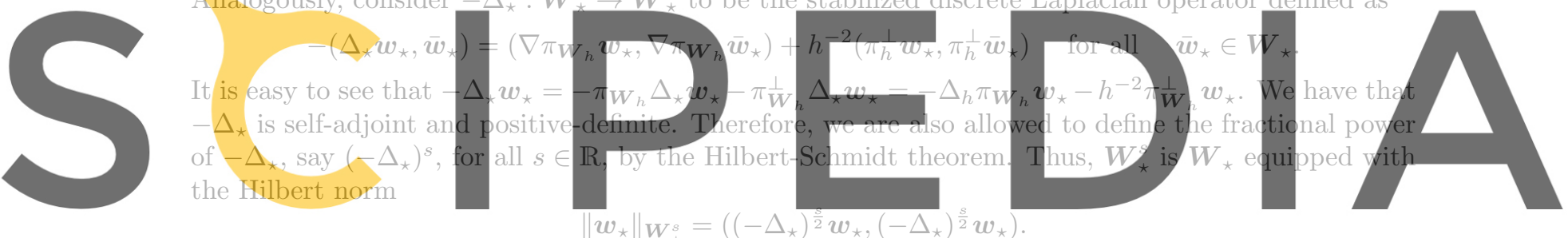

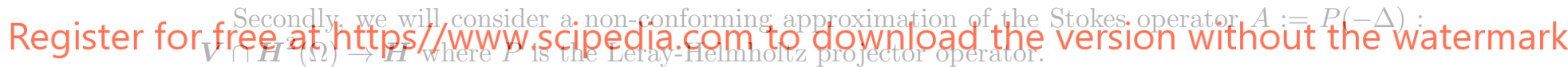

Let $A_{\star}: V_{\star} \rightarrow V_{\star}$ be defined as

$$
\left(A_{\star} \boldsymbol{v}_{\star}, \overline{\boldsymbol{v}}_{\star}\right)=\left(\nabla \pi_{\boldsymbol{W}_{h}} \boldsymbol{v}_{\star}, \nabla \pi_{\boldsymbol{W}_{h}} \overline{\boldsymbol{v}}_{\star}\right)+h^{-2}\left(\pi_{\boldsymbol{W}_{h}}^{\perp} \boldsymbol{v}_{\star}, \pi_{\boldsymbol{W}_{h}}^{\perp} \overline{\boldsymbol{v}}_{\star}\right) \quad \text { for all } \quad \overline{\boldsymbol{v}}_{\star} \in \overline{\boldsymbol{V}}_{\star} .
$$

Equivalently, one can write $A_{\star}=\pi_{\boldsymbol{W}_{h}} A_{\star}+\pi_{\boldsymbol{W}_{h}}^{\perp} A_{\star}:=A_{h}+\tilde{A}_{h}$ satisfying

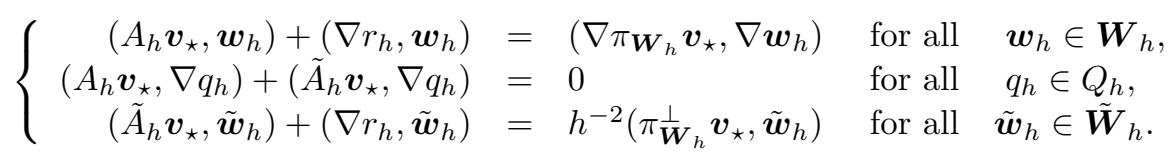

Again, $A_{\star}$ is a self-adjoint, positive-definite operator. Therefore, the fractional power of $A_{\star}$, say $A_{\star}^{s}$, is well-defined for all $s \in \mathbb{R}$. Moreover, $\boldsymbol{V}_{\star}^{s}$ denotes $\boldsymbol{V}_{\star}$ equipped with the Hilbert norm

$$
\left\|\boldsymbol{v}_{\star}\right\|_{\boldsymbol{V}_{\star}^{s}}=\left(A_{\star}^{\frac{s}{2}} \boldsymbol{v}_{\star}, A_{\star}^{\frac{s}{2}} \boldsymbol{v}_{\star}\right)^{\frac{1}{2}} .
$$

The family $\left\{\boldsymbol{V}_{\star}^{s}\right\}_{s \in \mathbb{R}}$ is a scale of Hilbert space with respect to the real method of interpolation.

Next we will consider a non-conforming approximation of the Leray-Helmholtz projection operator. Let $P_{\star}: \boldsymbol{L}^{2}(\Omega) \rightarrow \boldsymbol{V}_{\star}$ be defined as

$$
\left(P_{\star} \boldsymbol{v}, \overline{\boldsymbol{v}}_{\star}\right)=\left(\boldsymbol{v}, \overline{\boldsymbol{v}}_{\star}\right) \quad \text { for all } \quad \overline{\boldsymbol{v}}_{\star} \in \boldsymbol{V}_{\star} .
$$

Equivalently, one can write $P_{\star}=\pi_{\boldsymbol{W}_{h}} P_{\star}+\pi_{\boldsymbol{W}_{h}}^{\perp} P_{\star}:=P_{h}+\tilde{P}_{h}$ satisfying

$$
\left\{\begin{array}{rlll}
\left(P_{h} \boldsymbol{v}, \boldsymbol{w}_{h}\right)+\left(\nabla r_{h}, \boldsymbol{w}_{h}\right) & =\left(\pi_{\boldsymbol{W}_{h}} \boldsymbol{v}, \boldsymbol{w}_{h}\right) & \text { for all } & \boldsymbol{w}_{h} \in \boldsymbol{W}_{h} \\
\left(P_{h} \boldsymbol{v}, \nabla q_{h}\right)+\left(\tilde{P}_{h} \boldsymbol{v}, \nabla q_{h}\right) & =0 & \text { for all } & q_{h} \in Q_{h} \\
\left(\tilde{P}_{h} \boldsymbol{v}, \tilde{\boldsymbol{w}}_{h}\right)+\left(\nabla r_{h}, \tilde{\boldsymbol{w}}_{h}\right) & =\left(\pi_{\boldsymbol{W}_{h}}^{\perp} \boldsymbol{v}, \tilde{\boldsymbol{w}}_{h}\right) & \text { for all } & \tilde{\boldsymbol{w}}_{h} \in \tilde{\boldsymbol{W}}_{h} .
\end{array}\right.
$$


Finally, we define the stabilized Ritz projection operator onto $\boldsymbol{V}_{\star}$. Let $R_{\star}: \boldsymbol{H}_{0}^{1}(\Omega)=\pi_{\boldsymbol{W}_{h}} \boldsymbol{H}_{0}^{1} \oplus$ $\pi_{\boldsymbol{W}_{h}}^{\perp} \boldsymbol{H}_{0}^{1}(\Omega) \rightarrow \boldsymbol{V}_{\star}$ be defined as

$$
\left(\nabla \pi_{\boldsymbol{W}_{h}} R_{\star} \boldsymbol{v}, \nabla \pi_{\boldsymbol{W}_{h}} \boldsymbol{v}_{\star}\right)+h^{-2}\left(\pi_{\boldsymbol{W}_{h}}^{\perp} R_{\star} \boldsymbol{v}, \pi_{\boldsymbol{W}_{h}}^{\perp} \overline{\boldsymbol{v}}_{\star}\right)=\left(\nabla \pi_{\boldsymbol{W}_{h}} \boldsymbol{v}, \nabla \pi_{\boldsymbol{W}_{h}} \boldsymbol{v}_{\star}\right)+h^{-2}\left(\pi_{\boldsymbol{W}_{h}}^{\perp} \boldsymbol{v}_{,} \pi_{\boldsymbol{W}_{h}}^{\perp} \overline{\boldsymbol{v}}_{\star}\right),
$$

for all $\boldsymbol{v}_{\star} \in \boldsymbol{V}_{\star}$. Equivalently, one can write $R_{\star}=\pi_{\boldsymbol{W}_{h}} R_{\star}+\pi_{\boldsymbol{W}_{h}}^{\perp} R_{\star}:=R_{h}+\tilde{R}_{h}$ satisfying

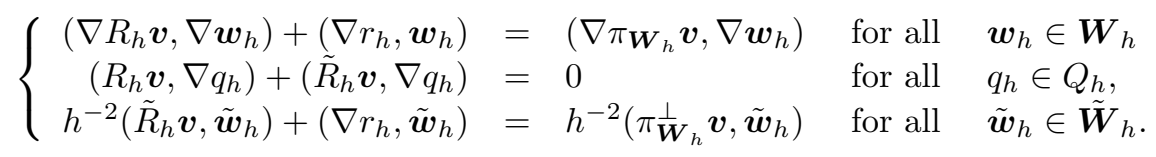

\section{TeChNicAl PRELIMinary RESUlts}

This section is mainly devote to some technical results concerning equivalence between norms and inf-sup conditions in fractional-order Sobolev spaces.

Lemma 4.1. Suppose that conditions (H1)-(H3) hold. Then there exist two positive constants c, C such that, for all $s \in \mathbb{R}$,

$$
c\left(\left\|\boldsymbol{w}_{h}\right\|_{W_{h}^{s}}+h^{-s}\left\|\tilde{\boldsymbol{w}}_{h}\right\|\right) \leq\left\|\boldsymbol{w}_{\star}\right\|_{W_{\star}^{s}} \leq C\left(\left\|\boldsymbol{w}_{h}\right\|_{W_{h}^{s}}+h^{-s}\left\|\tilde{\boldsymbol{w}}_{h}\right\|\right),
$$

for all $w_{\star}=w_{h}+\tilde{w}_{h} \in W_{\star}$.

Proof. The proof follows by observing that $\left(-\Delta_{\star} \boldsymbol{w}_{\star}\right)^{s}=\left(-\Delta \pi_{W_{h}} \boldsymbol{w}_{\star}\right)^{s}+h^{-s} \pi_{\boldsymbol{W}_{h}}^{\perp} \tilde{\boldsymbol{w}}_{\star}$ for all $\boldsymbol{w}_{\star}$
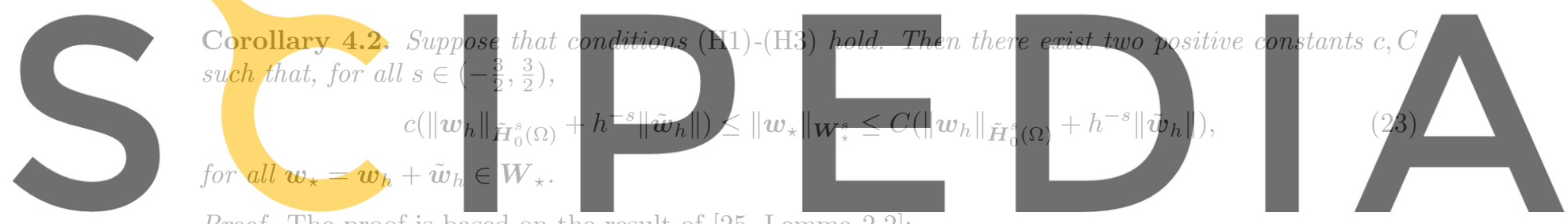

Proof. The proof is based on the result of [25, Lemma 2.2]:

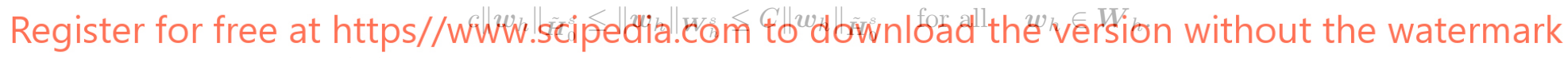
with $s \in\left(-\frac{3}{2}, \frac{3}{2}\right)$.

In the next lemma, we prove the stability of the stabilized discrete Leray-Helmholtz operator $P_{\star}=$ $P_{h}+\tilde{P}_{h}$.

Lemma 4.3. Assume that conditions (H1)-(H3) are satisfied. Then there exists a positive constant $C$, independent of $h$, such that, for all $s \in\left[0, \frac{1}{2}\right)$,

$$
\left\|P_{h} \boldsymbol{v}\right\|_{\tilde{\boldsymbol{H}}_{0}^{s}(\Omega)}+h^{-s}\left\|\tilde{P}_{h} \boldsymbol{v}\right\| \leq C\|\boldsymbol{v}\|_{\tilde{\boldsymbol{H}}_{0}^{s}(\Omega)} \quad \text { for all } \quad \boldsymbol{v} \in \tilde{\boldsymbol{H}}_{0}^{s}(\Omega),
$$

where $P_{\star}=P_{h}+\tilde{P}_{h}$ is the $\boldsymbol{L}^{2}(\Omega)$-orthogonal projection operator onto $\boldsymbol{V}_{\star}$.

Proof. Let $\boldsymbol{v} \in \tilde{\boldsymbol{H}}_{0}^{s}(\Omega)$. Then, by the Helmholtz-Hodge decomposition, there exists $r \in H_{\int=0}^{1}(\Omega)$ such that

$$
\boldsymbol{v}=P \boldsymbol{v}+\nabla r
$$

whose variational formulation reads as:

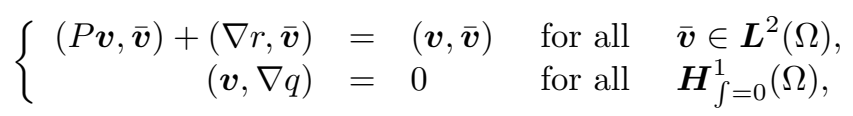

Note that problem (20) is the stabilized discrete counterpart of (25). From [16, Chapter II, Theorem 1.1], we get

$$
\left\|P_{\star} \boldsymbol{v}-P \boldsymbol{v}\right\|+\left\|\nabla r_{h}-\nabla r\right\| \leq C\left(\inf _{\boldsymbol{w}_{\star} \in \boldsymbol{W}_{\star}}\left\|P \boldsymbol{v}-\boldsymbol{w}_{\star}\right\|+\inf _{q_{h} \in Q_{h}}\left\|\nabla r-\nabla q_{h}\right\|\right) .
$$


Using the fact that $P_{\star}=P_{h}+\tilde{P}_{h}$ with $P_{h}$ and $\tilde{P}_{h}$ being $\boldsymbol{L}^{2}(\Omega)$-orthogonal by definition, we have

$$
\begin{aligned}
\left\|P_{\star} \boldsymbol{v}-P \boldsymbol{v}\right\|^{2} & =\left\|P_{h} \boldsymbol{v}-P \boldsymbol{v}\right\|^{2}+\left\|\tilde{P}_{h} \boldsymbol{v}\right\|^{2}-2\left(P \boldsymbol{v}, \tilde{P}_{h} \boldsymbol{v}\right) \\
& =\left\|P_{h} \boldsymbol{v}-P \boldsymbol{v}\right\|^{2}+\left\|\tilde{P}_{h} \boldsymbol{v}\right\|^{2}-2\left(P \boldsymbol{v}-\pi_{\boldsymbol{W}_{h}} P \boldsymbol{v}, \tilde{P}_{h} \boldsymbol{v}\right) \\
& \geq\left\|P_{h} \boldsymbol{v}-P \boldsymbol{v}\right\|^{2}+\left\|\tilde{P}_{h} \boldsymbol{v}\right\|^{2}-2\left\|P \boldsymbol{v}-\pi_{\boldsymbol{W}_{h}} P \boldsymbol{v}\right\|^{2}-\frac{1}{2}\left\|\tilde{P}_{h} \boldsymbol{v}\right\|^{2} \\
& =\left\|P_{h} \boldsymbol{v}-P \boldsymbol{v}\right\|^{2}+\frac{1}{2}\left\|\tilde{P}_{h} \boldsymbol{v}\right\|^{2}-2\left\|P \boldsymbol{v}-\pi_{\boldsymbol{W}_{h}} P \boldsymbol{v}\right\|^{2} .
\end{aligned}
$$

Inserting this back into (26), we obtain

$$
\left\|P_{h} \boldsymbol{v}-P \boldsymbol{v}\right\|+\left\|\nabla r_{h}-\nabla r\right\|+\left\|\tilde{P}_{h} \boldsymbol{v}\right\| \leq C\left(\inf _{\boldsymbol{w}_{h} \in \boldsymbol{W}_{h}}\left\|P \boldsymbol{v}-\boldsymbol{w}_{h}\right\|+\inf _{q_{h} \in Q_{h}}\left\|\nabla r-\nabla q_{h}\right\|\right) .
$$

From (11) and (12), we find that

$$
\left\|P_{h} \boldsymbol{v}-P \boldsymbol{v}\right\|+\left\|\tilde{P}_{h} \boldsymbol{v}\right\| \leq C h^{s}\|\boldsymbol{v}\|_{\tilde{\boldsymbol{H}}_{0}^{s}(\Omega)}
$$

and

$$
h^{-s}\left\|\tilde{P}_{h} \boldsymbol{v}\right\| \leq C\|\boldsymbol{v}\|_{\tilde{\boldsymbol{H}}_{0}^{s}(\Omega)} \cdot
$$

In view of (9), (10), (11) and (27), we write

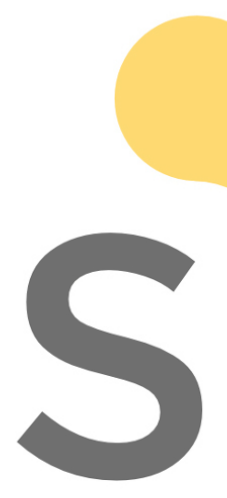

$\left\|P_{h} v\right\|_{\tilde{H}_{0}^{s}(\Omega)} \leq\left\|P_{h} v-\pi_{W_{h}} P v\right\|_{H_{0}^{s}(\Omega)}+\left\|\pi_{W_{h}} P_{h} v\right\|_{\tilde{H}_{0}^{s}(\Omega)}$

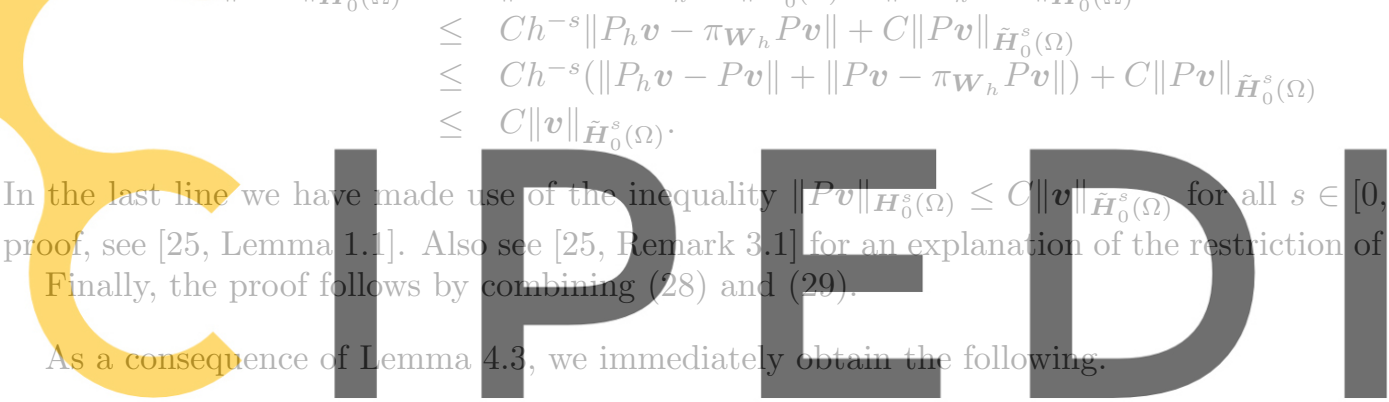

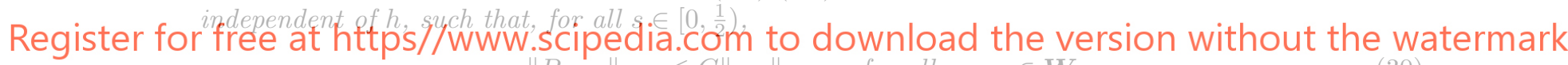

$$
\left\|P_{\star} w_{*}\right\|_{W_{*}^{s}} \leq C\left\|w_{*}\right\|_{W_{*}^{s}} \text { for all } w_{\star} \in W_{\star},
$$

where $P_{\star}=P_{h}+\tilde{P}_{h}$ is the $L^{2}(\Omega)$-orthogonal projection operator onto $V_{\star}$.

Proof. Let $\boldsymbol{w}_{\star} \in \boldsymbol{W}_{\star}$ such that $\boldsymbol{w}_{\star}=\boldsymbol{w}_{h}+\tilde{\boldsymbol{w}}_{h}$. Take $\boldsymbol{v}=\boldsymbol{w}_{h}$ in (24) to get

$$
\left\|P_{h} \boldsymbol{w}_{h}\right\|_{\tilde{\boldsymbol{H}}_{0}^{s}(\Omega)} \leq C\left\|\boldsymbol{w}_{h}\right\|_{\tilde{\boldsymbol{H}}_{0}^{s}(\Omega)} .
$$

Next, select $\boldsymbol{v}=\tilde{\boldsymbol{w}}_{h}$ in $(20)$. Now, pick $\boldsymbol{w}_{h}=P_{h} \tilde{\boldsymbol{w}}_{h}, q_{h}=r_{h}$, and $\tilde{\boldsymbol{w}}_{h}=\tilde{P}_{h} \tilde{\boldsymbol{w}}_{h}$ to obtain

$$
\left\|P_{h} \tilde{\boldsymbol{w}}_{h}\right\|^{2}+\frac{1}{2}\left\|\tilde{P}_{h} \tilde{\boldsymbol{w}}_{h}\right\|^{2} \leq \frac{1}{2}\left\|\tilde{\boldsymbol{w}}_{h}\right\|^{2} .
$$

In particular, we have

$$
h^{-s}\left\|\tilde{P}_{h} \tilde{\boldsymbol{w}}_{h}\right\|^{2} \leq h^{-s}\left\|\tilde{\boldsymbol{w}}_{h}\right\|^{2} .
$$

Combining (31) and (32), we prove (30) by Corollary 4.2 .

The following lemma sets up the equivalence between $\|\cdot\|_{\boldsymbol{W}_{\star}^{s}}$ and $\|\cdot\|_{\boldsymbol{V}_{\star}^{s}}$.

Lemma 4.5. Suppose that (H1)-(H2) are satisfied. There exist two positive constants $C, c$ such that, for each $s \in\left(-\frac{1}{2}, 2\right)$,

and, for each $s \in[-2,2]$,

$$
c\left\|\boldsymbol{v}_{\star}\right\|_{\boldsymbol{W}_{\star}^{s}} \leq\left\|\boldsymbol{v}_{\star}\right\|_{\boldsymbol{V}_{\star}^{s}} \quad \text { for all } \quad \boldsymbol{v}_{\star} \in \boldsymbol{V}_{\star},
$$

$$
\left\|\boldsymbol{v}_{\star}\right\|_{\boldsymbol{V}_{\star}^{s}} \leq C\left\|\boldsymbol{v}_{\star}\right\|_{\boldsymbol{W}_{\star}^{s}} \quad \text { for all } \quad \boldsymbol{v}_{\star} \in \boldsymbol{V}_{\star} .
$$


Proof. Assertion (33) is proved as follows. Take $\boldsymbol{v}_{\star} \in \boldsymbol{V}_{\star}$ such that $\boldsymbol{v}_{\star}=\boldsymbol{v}_{h}+\tilde{\boldsymbol{v}}_{h}$. Let $s \in[0,2]$. Define $(\boldsymbol{v}, r) \in\left(\boldsymbol{V} \cap \boldsymbol{H}_{0}^{1}(\Omega)\right) \times H_{\int=0}^{1}(\Omega)$ such that

$$
\left\{\begin{array}{rlrl}
-\Delta \boldsymbol{v}+\nabla r & =A_{\star} \boldsymbol{v}_{\star} & \text { in } & \Omega \\
\nabla \cdot \boldsymbol{v} & =0 & \text { in } \Omega .
\end{array}\right.
$$

By virtue of (H1), we have that $\|\Delta \boldsymbol{v}\|+\|\nabla r\| \leq C\left\|A_{\star} \boldsymbol{v}_{\star}\right\|$. Moreover, we have that $\left(\boldsymbol{v}_{h}, \tilde{\boldsymbol{v}}_{h}, r_{h}\right) \in$ $\boldsymbol{W}_{h} \times \tilde{\boldsymbol{W}}_{h} \times Q_{h}$ satisfies, for all $\left(\boldsymbol{w}_{h}, \tilde{\boldsymbol{w}}_{h}, q_{h}\right) \in \boldsymbol{W}_{h} \times \tilde{\boldsymbol{W}}_{h} \times Q_{h}$,

$$
\begin{aligned}
\left(\nabla \boldsymbol{v}_{h}, \nabla \boldsymbol{w}_{h}\right)+\left(\nabla r_{h}, \boldsymbol{w}_{h}\right) & =\left(A_{\star} \boldsymbol{v}_{\star}, \boldsymbol{w}_{h}\right), \\
\left(\boldsymbol{v}_{h}, \nabla q_{h}\right)+\left(\tilde{\boldsymbol{v}}_{h}, \nabla q_{h}\right) & =0, \\
h^{-2}\left(\tilde{\boldsymbol{v}}_{h}, \tilde{\boldsymbol{w}}_{h}\right)+\left(\nabla r_{h}, \tilde{\boldsymbol{w}}_{h}\right) & =\left(A_{\star} \boldsymbol{v}_{\star}, \tilde{\boldsymbol{w}}_{h}\right) .
\end{aligned}
$$

Comparing both problems, we get the following error estimates, that can be found in [3, Lemma 3.2 ]:

$$
\left\|\nabla\left(\boldsymbol{v}-\boldsymbol{v}_{h}\right)\right\|+h^{-1}\left\|\tilde{\boldsymbol{v}}_{h}\right\| \leq C h\left\|A_{\star} \boldsymbol{v}_{\star}\right\| .
$$

Next, let us write

$$
-\left(\Delta_{\star} \boldsymbol{v}_{\star}, \boldsymbol{w}_{\star}\right)=\left(\nabla\left(\pi_{\boldsymbol{W}_{h}} \boldsymbol{v}_{\star}-\boldsymbol{v}\right), \nabla \pi_{\boldsymbol{W}_{h}} \boldsymbol{w}_{\star}\right)+h^{-2}\left(\pi_{\boldsymbol{W}_{h}}^{\perp} \boldsymbol{v}_{\star}, \pi_{\boldsymbol{W}_{h}}^{\perp} \boldsymbol{w}_{\star}\right)-\left(\Delta \boldsymbol{v}, \boldsymbol{w}_{h}\right),
$$

where we have used the fact that $-\Delta_{\star} \boldsymbol{v}_{\star}=-\Delta_{h} \pi_{\boldsymbol{W}_{h}} \boldsymbol{v}_{\star}-h^{-2} \pi_{\boldsymbol{W}_{h}}^{\perp} \boldsymbol{v}_{\star}$. Select $\boldsymbol{w}_{\star}=-\Delta_{\star} \boldsymbol{v}_{\star}$ to find, from (9) and (35), that

$$
\begin{aligned}
& \left\|\Delta_{\star} \boldsymbol{v}_{\star}\right\|^{2}=\left\|\nabla\left(\boldsymbol{v}_{h}-\boldsymbol{v}\right)\right\|\left\|\nabla \pi_{\boldsymbol{W}_{h}} \Delta_{\star} \boldsymbol{v}_{\star}\right\|+h^{-2}\left\|\pi_{\boldsymbol{W}_{h}}^{\perp} \boldsymbol{v}_{\star}\right\|\left\|\pi_{\boldsymbol{W}_{h}}^{\perp} \Delta_{\star} \boldsymbol{v}_{\star}\right\|+\|\Delta \boldsymbol{v}\|\left\|\Delta_{\star} \boldsymbol{v}_{\star}\right\| \\
& \leq C\left\|A_{\star} \boldsymbol{v}_{\star}\right\|\left\|\Delta_{\star} \boldsymbol{v}_{\star}\right\|+C\left\|A_{\star} \boldsymbol{v}_{\star}\right\|\left\|\Delta_{\star} \boldsymbol{v}_{\star}\right\| \text {. }
\end{aligned}
$$

Thus, we obtain

$$
\left\|\Delta_{\star} \boldsymbol{v}_{\star}\right\| \leq C\left\|A_{\star} \boldsymbol{v}_{\star}\right\| .
$$

Equivalently,

$$
\left\|\boldsymbol{v}_{\star}\right\|_{\boldsymbol{W}_{\star}^{2}} \leq C\left\|\boldsymbol{v}_{\star}\right\|_{\boldsymbol{V}_{\star}^{2}} .
$$

We also have

$$
\left\|\boldsymbol{v}_{\star}\right\|_{\boldsymbol{W}_{\star}^{0}} \leq C\left\|\boldsymbol{v}_{\star}\right\|_{\boldsymbol{V}_{\star}^{0}} .
$$

By interpolation, one then deduces

$$
\left\|\boldsymbol{v}_{\star}\right\|_{\boldsymbol{W}_{\star}^{s}} \leq C\left\|\boldsymbol{v}_{\star}\right\|_{\boldsymbol{V}_{\star}^{s}} .
$$

for all $s \in[0,2]$.

Let $s \in\left(-\frac{1}{2}, 0\right]$. Then

$$
\left\|\boldsymbol{v}_{\star}\right\|_{\boldsymbol{W}_{\star}^{s}}=\sup _{\boldsymbol{w}_{\star} \in \boldsymbol{W}_{\star}^{-s} \backslash\{\mathbf{0}\}} \frac{\left(\boldsymbol{v}_{\star}, \boldsymbol{w}_{\star}\right)}{\left\|\boldsymbol{w}_{\star}\right\|_{\boldsymbol{W}_{\star}^{-s}}}=\sup _{\boldsymbol{w}_{\star} \in \boldsymbol{W}_{\star}^{-s} \backslash\{\mathbf{0}\}} \frac{\left(\boldsymbol{v}_{\star}, P_{\star} \boldsymbol{w}_{\star}\right)}{\left\|\boldsymbol{w}_{\star}\right\|_{\boldsymbol{W}_{\star}^{-s}}} .
$$

In view of (30), we have

$$
\begin{aligned}
&\left\|\boldsymbol{v}_{\star}\right\|_{\boldsymbol{W}_{\star}^{s}} \leq C \sup _{\boldsymbol{w}_{\star} \in \boldsymbol{W}_{\star}^{-s} \backslash\{\mathbf{0}\}} \frac{\left(\boldsymbol{v}_{\star}, P_{\star} \boldsymbol{w}_{\star}\right)}{\left\|P_{\star} \boldsymbol{w}_{\star}\right\|_{\boldsymbol{W}_{\star}^{-s}} \leq C \sup _{\boldsymbol{w}_{\star} \in \boldsymbol{V}_{\star}^{-s} \backslash\{\mathbf{0}\}} \frac{\left(\boldsymbol{v}_{\star}, \boldsymbol{w}_{\star}\right)}{\left\|\boldsymbol{w}_{\star}\right\|_{\boldsymbol{V}_{\star}^{-s}}}} \\
& \leq C\left\|\boldsymbol{v}_{\star}\right\|_{\boldsymbol{V}_{\star}^{s}} \sup _{\boldsymbol{w}_{\star} \in \boldsymbol{V}_{\star}^{-s} \backslash\{\mathbf{0}\}} \frac{\left\|\boldsymbol{w}_{\star}\right\|_{\boldsymbol{V}_{\star}^{-s}}}{\left\|\boldsymbol{w}_{\star}\right\|_{\boldsymbol{W}_{\star}^{-s}}} \leq C\left\|\boldsymbol{v}_{\star}\right\|_{\boldsymbol{V}_{\star}^{s} .} .
\end{aligned}
$$

Assertion (34) is proved as follows. Pick $\boldsymbol{v}_{\star} \in \boldsymbol{V}_{\star}$. Let $s \in[0,2]$. It should be first noted that $A_{\star} R_{\star} \boldsymbol{w}_{\star}=P_{\star} \Delta_{\star} \boldsymbol{w}_{\star}$ for all $\boldsymbol{w}_{\star} \in \boldsymbol{W}_{\star}$. Then, it follows that

$$
\left\|R_{\star} \boldsymbol{w}_{\star}\right\|_{\boldsymbol{V}_{\star}^{2}}=\left\|A_{\star} R_{\star} \boldsymbol{w}_{\star}\right\|=\left\|P_{\star} \Delta_{\star} \boldsymbol{w}_{\star}\right\| \leq\left\|\Delta_{\star} \boldsymbol{w}_{\star}\right\|=\left\|\boldsymbol{w}_{\star}\right\|_{\boldsymbol{W}_{\star}^{2}} .
$$

Next, we have that

$$
\left\|R_{\star} \boldsymbol{w}_{\star}\right\| \leq C\left\|\boldsymbol{w}_{\star}\right\|
$$

holds for all $\boldsymbol{w}_{\star} \in \boldsymbol{W}_{\star}$, i.e., $\left\|R_{\star} \boldsymbol{w}_{\star}\right\|_{\boldsymbol{V}_{\star}^{0}} \leq C\left\|\boldsymbol{w}_{h}\right\|_{\boldsymbol{W}_{h}^{0}}$ for all $\boldsymbol{w}_{\star} \in \boldsymbol{W}_{\star}$. Indeed, let $R_{\star}^{T}: \boldsymbol{V}_{\star} \rightarrow \boldsymbol{W}_{\star}$ the adjoint operator of $R_{\star}$, namely $R_{\star}^{T}=-\Delta_{\star} A_{\star}^{-1}$, since $R_{\star}=-A_{\star}^{-1} P_{\star} \Delta_{\star}$. It is clear that if

$$
\left\|R_{\star}^{T} \boldsymbol{v}_{\star}\right\| \leq C\left\|\boldsymbol{v}_{\star}\right\|
$$


holds for all $\boldsymbol{v}_{\star} \in \boldsymbol{V}_{\star}$, then (39) is true. But (40) is a consequence of (36). By interpolation between (38) and (39) for $\boldsymbol{w}_{\star}=\boldsymbol{v}_{\star}$, we obtain $\left\|\boldsymbol{v}_{\star}\right\|_{\boldsymbol{V}_{\star}^{s}} \leq C\left\|\boldsymbol{v}_{\star}\right\|_{\boldsymbol{W}_{\star}^{s}}$ for all $\boldsymbol{v}_{\star} \in \boldsymbol{V}_{\star}$ and $s \in[0,2]$.

Let $s \in[-2,0]$. From $(37)$, we have

$$
\left\|\boldsymbol{v}_{\star}\right\|_{\boldsymbol{V}_{\star}^{s}}=\sup _{\boldsymbol{v}_{\star} \in \boldsymbol{V} \star \backslash\{\mathbf{0}\}} \frac{\left(\boldsymbol{v}_{\star}, \overline{\boldsymbol{v}}_{\star}\right)}{\left\|\overline{\boldsymbol{v}}_{\star}\right\|_{\boldsymbol{V}_{\star}^{-s}}} \leq C \sup _{\boldsymbol{v}_{\star} \in \boldsymbol{V} \star \backslash\{\mathbf{0}\}} \frac{\left(\boldsymbol{v}_{\star}, \overline{\boldsymbol{v}}_{\star}\right)}{\left\|\overline{\boldsymbol{v}}_{\star}\right\|_{\boldsymbol{W}_{\star}^{-s}}} \leq\left\|\boldsymbol{v}_{\star}\right\|_{\boldsymbol{W}_{\star}^{s} .} .
$$

It completes the proof.

As a corollary to Lemma 4.5, we have the following inequality whose proof needs Corollary 4.2.

Corollary 4.6. Suppose that conditions (H1)-(H2) are satisfied. Then there exist two positive constants $C, c$ such that, for each $s \in\left(-\frac{1}{2}, \frac{3}{2}\right)$,

$$
c\left(\left\|\boldsymbol{v}_{h}\right\|_{\tilde{\boldsymbol{H}}_{0}^{s}(\Omega)}+h^{-s}\left\|\tilde{\boldsymbol{v}}_{h}\right\|\right) \leq\left\|\boldsymbol{v}_{\star}\right\|_{\boldsymbol{V}_{\star}^{s}} \quad \text { for all } \quad \boldsymbol{v}_{\star} \in \boldsymbol{V}_{\star},
$$

and, for each $s \in\left(-\frac{3}{2}, \frac{3}{2}\right)$,

$$
\left\|\boldsymbol{v}_{\star}\right\|_{\boldsymbol{V}_{\star}^{s}} \leq C\left(\left\|\boldsymbol{v}_{h}\right\|_{\tilde{\boldsymbol{H}}_{0}^{s}(\Omega)}+h^{-s}\left\|\tilde{\boldsymbol{v}}_{h}\right\|\right) \quad \text { for all } \quad \boldsymbol{v}_{\star} \in \boldsymbol{V}_{\star} .
$$

We are now concerned with the proof of an inf-sup condition in the framework of fractional Sobolev spaces.

Lemma 4.7. Under conditions (H1)-(H3), it follows that, for $s \in[0,1]$,

$$
\sup _{\boldsymbol{w}_{\star} \in \boldsymbol{W} \backslash\{\mathbf{0}\}} \frac{\left(\nabla q_{h}, \boldsymbol{w}_{\star}\right)}{\left\|\boldsymbol{w}_{\star}\right\|_{\boldsymbol{W}_{\star}^{1-s}}} \gtrsim\left\|q_{h}\right\|_{H^{s}(\Omega)},
$$

Proof. Let $q_{h} \in Q_{h}$. From (10) and (11), we have

$$
\begin{aligned}
\sup _{\boldsymbol{w}_{h} \in \boldsymbol{W}_{h} \backslash\{\mathbf{0}\}} \frac{\left(\nabla q_{h}, \boldsymbol{w}_{h}\right)}{\left\|\boldsymbol{w}_{h}\right\|_{\tilde{\boldsymbol{H}}_{0}^{1-s}(\Omega)}^{1-s}} & \geq \sup _{\boldsymbol{w} \in \tilde{\boldsymbol{H}}_{0}^{1-s}(\Omega) \backslash\{\mathbf{0}\}} \frac{\left(\nabla q_{h}, \pi_{\boldsymbol{W}_{h}} \boldsymbol{w}\right)}{\left\|\pi_{\boldsymbol{W}_{h}} \boldsymbol{w}\right\|_{\tilde{\boldsymbol{H}}_{0}^{1-s}(\Omega)}} \geq C_{\mathrm{st}}^{-1} \sup _{\boldsymbol{w} \in \tilde{\boldsymbol{H}}_{0}^{1-s}(\Omega) \backslash\{\mathbf{0}\}} \frac{\left(\nabla q_{h}, \pi_{\boldsymbol{W}_{h}} \boldsymbol{w}\right)}{\|\boldsymbol{w}\|_{\tilde{\boldsymbol{H}}_{0}^{1-s}(\Omega)}} \\
& \geq C_{\mathrm{st}}^{-1} \sup _{\boldsymbol{w} \in \tilde{\boldsymbol{H}}_{0}^{1-s} \backslash\{\mathbf{0}\}} \frac{\left(\nabla q_{h}, \boldsymbol{w}\right)}{\|\boldsymbol{w}\|_{\tilde{H}_{0}^{1-s}(\Omega)}}-C_{\mathrm{st}}^{-1} \sup _{\boldsymbol{w} \in \tilde{\boldsymbol{H}}_{0}^{s} \backslash\{\mathbf{0}\}} \frac{\left(\nabla q_{h}, \pi_{\boldsymbol{W}_{h}}^{\perp} \boldsymbol{w}\right)}{\left\|\pi_{\boldsymbol{W}_{h}} \boldsymbol{w}\right\|_{\tilde{\boldsymbol{H}}_{0}^{1-s}(\Omega)}^{1-s}} \\
& \geq C_{\mathrm{st}}^{-1}\left\|q_{h}\right\|_{H^{s}(\Omega)}-C_{\mathrm{st}}^{-1} C_{\mathrm{int}} h^{1-s}\left\|\nabla q_{h}\right\| .
\end{aligned}
$$

Moreover, we have, by (9),

$$
\sup _{\boldsymbol{w}_{h} \in \boldsymbol{W}_{h} \backslash\{\mathbf{0}\}} \frac{\left(\nabla q_{h}, \boldsymbol{w}_{h}\right)}{\left\|\boldsymbol{w}_{h}\right\|_{\tilde{\boldsymbol{H}}_{0}^{1-s}(\Omega)}} \geq \frac{\left\|\pi_{\boldsymbol{W}_{h}} \nabla q_{h}\right\|^{2}}{\left\|\pi_{\boldsymbol{W}_{h}} \nabla q_{h}\right\|_{\tilde{\boldsymbol{H}}_{0}^{1-s}(\Omega)}} \geq C_{\mathrm{inv}}^{-1} h^{1-s}\left\|\pi_{\boldsymbol{W}_{h}} \nabla q_{h}\right\| .
$$

Combining the previous two estimates, we get

$$
\sup _{\boldsymbol{w}_{h} \in \boldsymbol{W}_{h} \backslash\{\mathbf{0}\}} \frac{\left(\nabla q_{h}, \boldsymbol{w}_{h}\right)}{\left\|\boldsymbol{w}_{h}\right\|_{\tilde{\boldsymbol{H}}_{0}^{1-s}(\Omega)}} \geq C_{1}\left\|q_{h}\right\|_{H^{s}(\Omega)}-C_{2} h^{1-s}\left\|\pi_{\boldsymbol{W}_{h}}^{\perp} \nabla q_{h}\right\|,
$$

where $C_{1}^{-1}=C_{2}^{-1} C_{\text {int }}$ and $C_{2}^{-1}=\left(C_{\text {st }}+C_{\text {int }} C_{\text {inv }}\right)$. Since $\pi_{\boldsymbol{W}_{h}}^{\perp}\left(\nabla Q_{h}\right) \subset \tilde{\boldsymbol{W}}_{h}$, we find that

$$
\sup _{\tilde{\boldsymbol{w}}_{h} \in \tilde{\boldsymbol{W}}_{h} \backslash\{\mathbf{0}\}} \frac{\left(\nabla q_{h}, \tilde{\boldsymbol{w}}_{h}\right)}{h^{s-1}\left\|\tilde{\boldsymbol{w}}_{h}\right\|} \geq h^{1-s}\left\|\pi_{\boldsymbol{W}_{h}}^{\perp} \nabla q_{h}\right\| .
$$

At this point, we have proved that

$$
\sup _{\boldsymbol{w}_{h} \in \boldsymbol{W}_{h} \backslash\{\mathbf{0}\}} \frac{\left(\nabla q_{h}, \boldsymbol{w}_{h}\right)}{\left\|\boldsymbol{w}_{h}\right\|_{\tilde{\boldsymbol{H}}_{0}^{1-s}(\Omega)}^{1-}}+C_{2} \sup _{\tilde{\boldsymbol{w}}_{h} \in \tilde{\boldsymbol{W}}_{h} \backslash\{\mathbf{0}\}} \frac{\left(\nabla q_{h}, \tilde{\boldsymbol{w}}_{h}\right)}{h^{s-1}\left\|\tilde{\boldsymbol{w}}_{h}\right\|} \geq C_{1}\left\|q_{h}\right\|_{H^{s}(\Omega)} .
$$

Finally, observe, by (22), that

$$
\sup _{\boldsymbol{w}_{\star} \in \boldsymbol{W} \star\{\mathbf{0}\}} \frac{\left(\nabla q_{h}, \boldsymbol{w}_{\star}\right)}{\left\|\boldsymbol{w}_{\star}\right\|_{\boldsymbol{W}_{\star}^{1-s}}} \geq \sup _{\boldsymbol{w}_{h} \in \boldsymbol{W}_{h} \backslash\{\mathbf{0}\}} \frac{\left(\nabla q_{h}, \boldsymbol{w}_{h}\right)}{\left\|\tilde{\boldsymbol{w}}_{h}\right\|_{\tilde{\boldsymbol{H}}_{0}^{1-s}(\Omega)}}
$$


and

Then it follows that

$$
\sup _{\boldsymbol{w}_{\star} \in \boldsymbol{W}_{\star} \backslash\{\mathbf{0}\}} \frac{\left(\nabla q_{h}, \boldsymbol{w}_{\star}\right)}{\left\|\boldsymbol{w}_{\star}\right\|_{\boldsymbol{W}_{\star}^{1-s}}} \geq \sup _{\tilde{\boldsymbol{w}}_{h} \in \tilde{\boldsymbol{W}}_{h} \backslash\{\mathbf{0}\}} \frac{\left(\nabla q_{h}, \tilde{\boldsymbol{w}}_{h}\right)}{h^{s-1}\left\|\boldsymbol{w}_{h}\right\|}
$$

$$
\left(1+C_{2}\right) \sup _{\boldsymbol{w}_{\star} \in \boldsymbol{W}_{\star} \backslash\{\mathbf{0}\}} \frac{\left(\nabla q_{h}, \boldsymbol{w}_{\star}\right)}{\left\|\boldsymbol{w}_{\star}\right\|_{\boldsymbol{W}_{\star}^{1-s}}} \geq C_{1}\left\|q_{h}\right\|_{H^{s}(\Omega)}
$$

or equivalently, for any $q_{h} \in Q_{h}$, there exists an element $\boldsymbol{w}_{\star} \in \boldsymbol{W}_{\star}$ with norm $\left\|\boldsymbol{w}_{\star}\right\|_{\boldsymbol{W}_{\star}^{1-s}}=1$, such that $\left(\nabla q_{h}, v_{\star}\right) \geq C\left\|q_{h}\right\|_{H^{s}(\Omega)}$. It easily proves the lemma.

\section{A PRIORI ENERGY ESTIMATES}

In this section we derive a series of a priori energy estimates resulting in that the sequence of the approximate solutions $\left(\boldsymbol{u}_{h}, p_{h}\right)$ to scheme (17) approaches to a weak solution and a suitable weak solution in Section 6.

Theorem 5.1. Under assumptions (H1), (H2), and (H4), there is a positive constant C, independent of $h$, such that

$$
\left\|\boldsymbol{u}_{h}\right\|_{L^{\infty}\left(0, T ; \boldsymbol{L}^{2}(\Omega)\right) \cap L^{2}\left(0, T ; \boldsymbol{H}_{0}^{1}(\Omega)\right)}+\left\|\tilde{\boldsymbol{u}}_{h}\right\|_{L^{\infty}\left(0, T ; \boldsymbol{L}^{2}(\Omega)\right) \cap \tau^{-1} L^{2}\left(0, T ; \boldsymbol{L}^{2}(\Omega)\right)} \leq C .
$$

Proof. Take $\boldsymbol{v}_{h}=\boldsymbol{u}_{h}$ in (17a), $\tilde{\boldsymbol{v}}_{h}=\tilde{\boldsymbol{u}}_{h}$ in (17c) and $q_{h}=p_{h}$ in (17b) to get

$$
\frac{1}{2} \frac{\mathrm{d}}{\mathrm{d} t}\left(\left\|\boldsymbol{u}_{h}\right\|^{2}+\left\|\tilde{\boldsymbol{u}}_{h}\right\|^{2}\right)+\nu\left\|\nabla \boldsymbol{u}_{h}\right\|^{2}+\tau^{-1}\left\|\tilde{\boldsymbol{u}}_{h}\right\|^{2}=\left(\boldsymbol{f}_{h}, \boldsymbol{u}_{h}\right) .
$$

Next we estimate the term $\left(\boldsymbol{f}_{h}, \boldsymbol{u}_{h}\right)$. Thus, we have

$$
\left(\boldsymbol{f}_{h}, \boldsymbol{u}_{h}\right) \leq\|\boldsymbol{f}\|_{\boldsymbol{H}^{-1}(\Omega)}\left\|\nabla \boldsymbol{u}_{h}\right\| \leq \frac{1}{2 \nu}\|\boldsymbol{f}\|_{\boldsymbol{H}^{-1}(\Omega)}^{2}+\frac{\nu}{2}\left\|\nabla \boldsymbol{u}_{h}\right\|^{2} .
$$

Therefore, we obtain

$$
\frac{\mathrm{d}}{\mathrm{d} t}\left(\left\|\boldsymbol{u}_{h}\right\|^{2}+\left\|\tilde{\boldsymbol{u}}_{h}\right\|^{2}\right)+\nu\left\|\nabla \boldsymbol{u}_{h}\right\|^{2}+\tau^{-1}\left\|\tilde{\boldsymbol{u}}_{h}\right\|^{2} \leq \frac{1}{\nu}\|\boldsymbol{f}\|_{\boldsymbol{H}^{-1}(\Omega)}^{2},
$$

which, integrated over $(0, t)$, leads to the desired result.

Remark 5.2. From (43), we have

$$
\left\|\boldsymbol{u}_{h}\right\|_{L^{r}\left(0, T ; \boldsymbol{H}^{2 / r}(\Omega)\right)}+\left\|\boldsymbol{u}_{h}\right\|_{L^{r}\left(0, T ; \boldsymbol{L}^{k}(\Omega)\right)} \leq C,
$$

where $\frac{3}{k}+\frac{2}{r}=\frac{3}{2}$ with $r \in[2, \infty]$ and $k \in[2,6]$. The proof is based on the interpolation inequality between $\boldsymbol{L}^{2}(\Omega)$ and $\boldsymbol{H}^{1}(\Omega)$, i.e., $\|\boldsymbol{w}\|_{\boldsymbol{H}^{\frac{2}{r}(\Omega)}} \leq C\|\boldsymbol{w}\|_{\boldsymbol{L}^{2}(\Omega)}^{1-\frac{2}{r}}\|\boldsymbol{w}\|_{\boldsymbol{H}^{1}(\Omega)}^{\frac{2}{2}}$ for $r \in[2, \infty]$, and the Sobolev embedding $\boldsymbol{H}^{\frac{2}{r}}(\Omega) \hookrightarrow \boldsymbol{L}^{k}(\Omega)$ for $\frac{1}{k}=\frac{1}{2}-\frac{2}{3 r}$ and $r>\frac{4}{3}$.

Consider $\boldsymbol{u}_{\star}=\boldsymbol{u}_{h}+\tilde{\boldsymbol{u}}_{h}$. Then problem (17) reads as follows. Find $\boldsymbol{u}_{\star} \in H^{1}\left(0, T ; \boldsymbol{W}_{\star}\right)$ and $p_{h} \in L^{2}\left(0, T ; Q_{h}\right)$ such that

$$
\left(\partial_{t} \boldsymbol{u}_{\star}, \boldsymbol{w}_{\star}\right)+b_{\star}\left(\boldsymbol{u}_{\star}, \boldsymbol{u}_{\star}, \boldsymbol{w}_{\star}\right)-\nu\left(\Delta_{\star} \boldsymbol{u}_{\star}, \boldsymbol{w}_{\star}\right)-\left(\nabla p_{h}, \boldsymbol{w}_{\star}\right)=\left(\boldsymbol{f}_{h}, \pi_{\boldsymbol{W}_{h}}\left(\boldsymbol{w}_{\star}\right)\right),
$$

for all $\boldsymbol{w}_{\star} \in \boldsymbol{W}_{\star}$, with the initial condition $\boldsymbol{u}_{\star}(0)=\boldsymbol{u}_{0 h}+\tilde{\boldsymbol{u}}_{0 h}$, where

$$
b_{\star}\left(\boldsymbol{u}_{\star}, \boldsymbol{v}_{\star}, \boldsymbol{w}_{\star}\right)=b\left(\pi_{\boldsymbol{W}_{h}} \boldsymbol{u}_{\star}, \pi_{\boldsymbol{W}_{h}} \boldsymbol{u}_{\star}, \boldsymbol{w}_{\star}\right)-b\left(\pi_{\boldsymbol{W}_{h}} \boldsymbol{u}_{\star}, \pi_{\boldsymbol{W}_{h}} \boldsymbol{w}_{\star}, \pi_{\boldsymbol{W}_{h}}^{\perp} \boldsymbol{u}_{\star}\right)+\tau_{\infty}^{-1}\left(\pi_{\boldsymbol{W}_{h}}^{\perp} \boldsymbol{u}_{\star}, \pi_{\boldsymbol{W}_{h}}^{\perp} \boldsymbol{w}_{\star}\right)
$$

with $\tau_{\infty}^{-1}=C_{c} \frac{\left\|\pi_{\boldsymbol{W}_{h}} \boldsymbol{u}_{\star}\right\|_{L} \infty(\Omega)}{h}$. We now define the operator $\mathcal{N}_{\star}: \boldsymbol{W}_{\star} \times \boldsymbol{W}_{\star} \rightarrow \boldsymbol{W}_{\star}^{-1}$ via duality by the formula

$$
\left\langle\mathcal{N}_{\star}\left(\boldsymbol{u}_{\star}, \boldsymbol{v}_{\star}\right), \boldsymbol{w}_{\star}\right\rangle=b_{\star}\left(\boldsymbol{u}_{\star}, \boldsymbol{v}_{\star}, \boldsymbol{w}_{\star}\right) .
$$

Lemma 5.3. Suppose that assumptions (H1)-(H3) hold. Let $q$ be as in $(\mathrm{H} 4)$, but $q \in\left[1, \frac{3}{2}\right)$, and let $s$ be as in $(\mathrm{C})$, i.e, $s \in\left[\frac{1}{2}, \frac{3}{2}\right)$. Then it follows that

$$
\left\|\mathcal{N}_{\star}\left(\boldsymbol{u}_{\star}, \boldsymbol{u}_{\star}\right)\right\|_{\boldsymbol{W}_{\star}^{-s}} \leq C\left\|\pi_{\boldsymbol{W}_{h}} \boldsymbol{u}_{\star}\right\|_{\boldsymbol{L}^{k}(\Omega)}\left(\left\|\nabla \pi_{\boldsymbol{W}_{h}} \boldsymbol{u}_{\star}\right\|+\tau^{-\frac{1}{2}}\left\|\pi_{\boldsymbol{W}_{h}}^{\perp} \boldsymbol{u}_{\star}\right\|\right)
$$

with $\frac{1}{k}+\frac{1}{2}=\frac{1}{q}$. 
Proof. By definition of $b(\cdot, \cdot, \cdot)$, we have

$$
b\left(\pi_{\boldsymbol{W}_{h}} \boldsymbol{u}_{\star}, \pi_{\boldsymbol{W}_{h}} \boldsymbol{u}_{\star}, \boldsymbol{w}_{\star}\right)=\left\langle\mathcal{N}\left(\pi_{\boldsymbol{W}_{h}} \boldsymbol{u}_{\star}, \pi_{\boldsymbol{W}_{h}} \boldsymbol{u}_{\star}\right), \boldsymbol{w}_{\star}\right\rangle \leq\left\|\mathcal{N}\left(\pi_{\boldsymbol{W}_{h}} \boldsymbol{u}_{\star}, \pi_{\boldsymbol{W}_{h}} \boldsymbol{u}_{\star}\right)\right\|_{\boldsymbol{W}_{\star}^{-s}}\left\|\boldsymbol{w}_{\star}\right\|_{\boldsymbol{W}_{\star}^{s}},
$$

which, combined with (22), gives

$$
\left\|\mathcal{N}\left(\pi_{\boldsymbol{W}_{h}} \boldsymbol{u}_{\star}, \pi_{\boldsymbol{W}_{h}} \boldsymbol{u}_{\star}\right)\right\|_{\boldsymbol{W}_{\star}^{-s}} \leq C\left\|\mathcal{N}\left(\pi_{\boldsymbol{W}_{h}} \boldsymbol{u}_{\star}, \pi_{\boldsymbol{W}_{h}} \boldsymbol{u}_{\star}\right)\right\|_{\tilde{\boldsymbol{H}}_{0}^{-s}(\Omega)}+C h^{s}\left\|\mathcal{N}\left(\pi_{\boldsymbol{W}_{h}} \boldsymbol{u}_{\star}, \pi_{\boldsymbol{W}_{h}} \boldsymbol{u}_{\star}\right)\right\| .
$$

From the continuous embedding $\tilde{\boldsymbol{H}}_{0}^{s}(\Omega) \hookrightarrow \boldsymbol{L}^{q^{\prime}}(\Omega)$, with $\frac{1}{q^{\prime}}+\frac{1}{q}=1$, we bound

$$
\left\|\mathcal{N}\left(\pi_{\boldsymbol{W}_{h}} \boldsymbol{u}_{\star}, \pi_{\boldsymbol{W}_{h}}\left(\boldsymbol{u}_{\star}\right)\right)\right\|_{\boldsymbol{W}_{\star}^{-s}} \leq C\left\|\mathcal{N}_{h}\left(\pi_{\boldsymbol{W}_{h}} \boldsymbol{u}_{\star}, \pi_{\boldsymbol{W}_{h}} \boldsymbol{u}_{\star}\right)\right\|_{\boldsymbol{L}^{q}(\Omega)}+h^{s}\left\|\mathcal{N}\left(\pi_{\boldsymbol{W}_{h}} \boldsymbol{u}_{\star}, \pi_{\boldsymbol{W}_{h}} \boldsymbol{u}_{\star}\right)\right\| .
$$

Next, using (6), the definition of $s$, and the relation $\frac{1}{k}+\frac{1}{2}=\frac{1}{q}$ gives

$$
\left\|\mathcal{N}\left(\pi_{\boldsymbol{W}_{h}} \boldsymbol{u}_{\star}, \pi_{\boldsymbol{W}_{h}} \boldsymbol{u}_{\star}\right)\right\|_{\boldsymbol{L}^{q}(\Omega)} \leq\left\|\nabla \pi_{\boldsymbol{W}_{h}} \boldsymbol{u}_{\star}\right\|\left\|\pi_{\boldsymbol{W}_{h}} \boldsymbol{u}_{\star}\right\|_{\boldsymbol{L}^{k}(\Omega)},
$$

and

$$
h^{s}\left\|\mathcal{N}_{h}\left(\pi_{\boldsymbol{W}_{h}} \boldsymbol{u}_{\star}, \pi_{\boldsymbol{W}_{h}} \boldsymbol{u}_{\star}\right)\right\| \leq C h^{s}\left\|\pi_{\boldsymbol{W}_{h}} \boldsymbol{u}_{\star}\right\|_{\boldsymbol{L}^{\infty}(\Omega)}\left\|\nabla \pi_{\boldsymbol{W}_{h}} \boldsymbol{u}_{\star}\right\| \leq C\left\|\nabla \pi_{\boldsymbol{W}_{h}} \boldsymbol{u}_{\star}\right\|\left\|\pi_{\boldsymbol{W}_{h}} \boldsymbol{u}_{\star}\right\|_{\boldsymbol{L}^{k}(\Omega)},
$$

which imply that

$$
b\left(\pi_{\boldsymbol{W}_{h}} \boldsymbol{u}_{\star}, \pi_{\boldsymbol{W}_{h}} \boldsymbol{u}_{\star}, \boldsymbol{w}_{\star}\right) \leq\left\|\nabla \pi_{\boldsymbol{W}_{h}} \boldsymbol{u}_{\star}\right\|\left\|\pi_{\boldsymbol{W}_{h}} \boldsymbol{u}_{\star}\right\|_{\boldsymbol{L}^{k}(\Omega)}\left\|\boldsymbol{w}_{\star}\right\|_{\boldsymbol{W}_{\star}^{s}} .
$$

Next, for $s \in\left[\frac{1}{2}, 1\right]$, we write

$$
\begin{aligned}
b\left(\pi_{\boldsymbol{W}_{h}} \boldsymbol{u}_{\star}, \pi_{\boldsymbol{W}_{h}} \boldsymbol{w}_{\star}, \pi_{\boldsymbol{W}_{h}}^{\perp} \boldsymbol{u}_{\star}\right)= & \left(\left(\pi_{\boldsymbol{W}_{h}} \boldsymbol{u}_{\star} \cdot \nabla\right) \pi_{\boldsymbol{W}_{h}} \boldsymbol{w}_{\star}, \pi_{\boldsymbol{W}_{h}}^{\perp} \boldsymbol{u}_{\star}\right) \\
& +\frac{1}{2}\left(\nabla \cdot \pi_{\boldsymbol{W}_{h}} \boldsymbol{u}_{\star} \pi_{\boldsymbol{W}_{h}} \boldsymbol{w}_{\star}, \pi_{\boldsymbol{W}_{h}}^{\perp} \boldsymbol{u}_{\star}\right) \\
\leq & \left\|\pi_{\boldsymbol{W}_{h}} \boldsymbol{u}_{\star}\right\|_{\boldsymbol{L}^{\infty}(\Omega)}\left\|\nabla \pi_{\boldsymbol{W}_{h}} \boldsymbol{w}_{\star}\right\|\left\|\pi_{\boldsymbol{W}_{h}}^{\perp} \boldsymbol{u}_{\star}\right\| \\
& +\frac{1}{2}\left\|\nabla \cdot \pi_{\boldsymbol{W}_{h}} \boldsymbol{u}_{\star}\right\|_{\boldsymbol{L}^{k}(\Omega)}\left\|\pi_{\boldsymbol{W}_{h}} \boldsymbol{w}_{\star}\right\|_{\boldsymbol{L}^{\infty}(\Omega)}\left\|\pi_{\boldsymbol{W}_{h}}^{\perp} \boldsymbol{u}_{\star}\right\| \\
\leq & C h^{-\frac{3}{k}}\left\|\pi_{\boldsymbol{W}_{h}} \boldsymbol{u}_{\star}\right\|_{\boldsymbol{L}^{k}(\Omega)} h^{-1+s}\left\|\pi_{\boldsymbol{W}_{h}} \boldsymbol{w}_{\star}\right\|_{\tilde{\boldsymbol{H}}_{0}^{s}(\Omega)}^{s}\left\|\pi_{\boldsymbol{W}_{h}}^{\perp} \boldsymbol{u}_{\star}\right\| \\
& +C h^{-1}\left\|\pi_{\boldsymbol{W}_{h}} \boldsymbol{u}_{\star}\right\|_{\boldsymbol{L}^{k}(\Omega)}\left\|\pi_{\boldsymbol{W}_{h}} \boldsymbol{w}_{\star}\right\|_{\boldsymbol{L}^{q^{\prime}}(\Omega)}\left\|\pi_{\boldsymbol{W}_{h}}^{\perp} \boldsymbol{u}_{\star}\right\| \\
\leq & C\left\|\pi_{\boldsymbol{W}_{h}} \boldsymbol{u}_{\star}\right\|_{\boldsymbol{L}^{k}(\Omega)} \tau^{-\frac{1}{2}}\left\|\pi_{\boldsymbol{W}_{h}}^{\perp} \boldsymbol{u}_{\star}\right\|\left\|\pi_{\boldsymbol{W}_{h}} \boldsymbol{w}_{\star}\right\|_{\tilde{\boldsymbol{H}}_{0}^{s}(\Omega)}^{s} \\
\leq & \left\|\pi_{\boldsymbol{W}_{h}} \boldsymbol{u}_{\star}\right\|_{\boldsymbol{L}^{k}(\Omega)} \tau^{-\frac{1}{2}}\left\|\pi_{\boldsymbol{W}_{h}}^{\perp} \boldsymbol{u}_{\star}\right\|\left\|\boldsymbol{w}_{\star}\right\|_{\boldsymbol{W}_{\star}^{s}},
\end{aligned}
$$

where we have utilized (6) and (8), the relation $\frac{1}{k}+\frac{1}{2}=\frac{1}{q}$ and the definition of $s$. Moreover, we have also utilized $\left\|\pi_{\boldsymbol{W}_{h}} \boldsymbol{u}_{\star}\right\|_{\boldsymbol{L}^{q^{\prime}}(\Omega)} \leq C\left\|\pi_{\boldsymbol{W}_{h}} \boldsymbol{u}_{\star}\right\|_{\tilde{\boldsymbol{H}}_{0}^{s}(\Omega)}, q^{\prime} \in[3, \infty]$, and the relation (23). The estimate for $s \in\left(1, \frac{3}{2}\right)$ follows readily from applying the above arguments and the continuous embedding $\tilde{\boldsymbol{H}}_{0}^{s}(\Omega) \hookrightarrow$ $\boldsymbol{H}_{0}^{1}(\Omega)$.

Finally,

$$
\begin{aligned}
\tau_{\infty}^{-1}\left(\pi_{\boldsymbol{W}_{h}}^{\perp} \boldsymbol{u}_{\star}, \pi_{\boldsymbol{W}_{h}}^{\perp} \boldsymbol{w}_{\star}\right) & \leq \tau_{\infty}^{-1}\left\|\pi_{\boldsymbol{W}_{h}}^{\perp} \boldsymbol{u}_{\star}\right\|\left\|\pi_{\boldsymbol{W}_{h}}^{\perp} \boldsymbol{u}_{\star}\right\| \\
& =C_{C} h^{-1}\left\|\pi_{\boldsymbol{W}_{h}} \boldsymbol{u}_{\star}\right\|_{\boldsymbol{L}^{\infty}(\Omega)}\left\|\pi_{\boldsymbol{W}_{h}}^{\perp} \boldsymbol{u}_{\star}\right\|\left\|\pi_{\boldsymbol{W}_{h}}^{\perp} \boldsymbol{w}_{\star}\right\| \\
& \leq C\left\|\pi_{\boldsymbol{W}_{h}} \boldsymbol{u}_{\star}\right\|_{\boldsymbol{L}^{k}(\Omega)}\left\|\pi_{\boldsymbol{W}_{h}}^{\perp} \boldsymbol{u}_{\star}\right\| h^{-s}\left\|\pi_{\boldsymbol{W}_{h}}^{\perp} \boldsymbol{w}_{\star}\right\| .
\end{aligned}
$$

A duality argument shows then that

$$
\left\|\mathcal{N}_{\star}\left(\boldsymbol{u}_{\star}, \boldsymbol{u}_{\star}\right)\right\|_{\boldsymbol{W}_{\star}^{-s}} \leq C\left(\tau^{-\frac{1}{2}}\left\|\pi_{\boldsymbol{W}_{h}}^{\perp} \boldsymbol{u}_{\star}\right\|+\left\|\nabla \pi_{\boldsymbol{W}_{h}} \boldsymbol{u}_{\star}\right\|\right)\left\|\pi_{\boldsymbol{W}_{h}} \boldsymbol{u}_{\star}\right\|_{\boldsymbol{L}^{k}(\Omega)} .
$$

Corollary 5.4. Suppose that assumptions (H1)-(H3) hold. Let $p$ be as in $(\mathrm{H} 4)$, but $p \in[1,2)$, and $s$ be as in $(\mathrm{C})$, but $s \in\left[\frac{1}{2}, \frac{3}{2}\right)$. Then there exists $C>0$, independent of $h$, such that

$$
\left\|\mathcal{N}_{\star}\left(\boldsymbol{u}_{\star}, \boldsymbol{u}_{\star}\right)\right\|_{L^{p}\left(0, T ; \boldsymbol{W}_{\star}^{-s}\right)} \leq C
$$

Proof. The proof follows by using the $L^{r}\left(0, T ; \boldsymbol{L}^{k}(\Omega)\right)$ estimate stated in (44).

Lemma 5.5. Suppose that (H1)-(H3) hold. Let $s$ and $\bar{r}$ be as in (C), but $s \in\left[\frac{1}{2}, \frac{3}{2}\right)$ and $\bar{r} \in\left[0, \frac{1}{2}\right)$. Then there exists $C>0$, independent of $h$, such that

$$
\left\|\mathcal{N}_{\star}\left(\boldsymbol{u}_{\star}, \boldsymbol{u}_{\star}\right)\right\|_{H^{-r}\left(\mathbb{R} ; \boldsymbol{W}_{\star}^{-s}\right)} \leq C
$$

with $r>\bar{r}$. 
Proof. Let $\mathfrak{N}_{\star}$ be the extension of $\mathcal{N}_{\star}$ by zero off $[0, T]$. We have, by the Hausdorff-Young inequality, that

$$
\begin{aligned}
\left\|\mathfrak{N}_{\star}\left(\boldsymbol{u}_{\star}, \boldsymbol{u}_{\star}\right)\right\|_{H^{-r}\left(\mathbb{R} ; \boldsymbol{W}_{\star}^{-s}\right)} & =\int_{\mathbb{R}}(1+|\xi|)^{-2 r}\left\|\mathcal{F} \mathfrak{N}_{\star}\left(\boldsymbol{u}_{\star}, \boldsymbol{u}_{\star}\right)\right\|_{\boldsymbol{W}_{\star}^{-s}}^{2} d \xi \\
& \leq\left\|(1+|\xi|)^{-2 r}\right\|_{L^{\frac{1}{2 r}}(\mathbb{R})}\left\|\mathcal{F} \mathfrak{N}_{\star}\left(\boldsymbol{u}_{\star}, \boldsymbol{u}_{\star}\right)\right\|_{L^{p^{\prime}}\left(\mathbb{R} ; \boldsymbol{W}_{\star}^{-s}\right)}^{2} . \\
& \leq C\left\|\mathfrak{N}_{\star}\left(\boldsymbol{u}_{\star}, \boldsymbol{u}_{\star}\right)\right\|_{L^{p}\left(0, T ; \boldsymbol{W}_{\star}^{-s}\right)} .
\end{aligned}
$$

Observe that $\left\|(1+|\xi|)^{-2 r}\right\|_{L^{\frac{1}{2 \bar{r}}(\mathbb{R})}}=\left\|(1+|\xi|)^{-\frac{r}{\bar{r}}}\right\|_{L^{1}(\mathbb{R})}<\infty$ if $\frac{r}{\bar{r}}>1$. In particular, it holds for $r>\bar{r}$. It completes the proof.

Before proceeding with a priori energy estimates for $\partial_{t} \boldsymbol{u}_{h}$ and $\partial_{t} \tilde{\boldsymbol{u}}_{h}$, let us write (45) as a nonlinear heat equation

where

$$
\partial_{t} \boldsymbol{u}_{\star}+\nu A_{\star} \boldsymbol{u}_{\star}=P_{\star} \boldsymbol{g}
$$

$$
\boldsymbol{g}:=\boldsymbol{f}_{h}-\mathcal{N}_{\star}\left(\boldsymbol{u}_{\star}, \boldsymbol{u}_{\star}\right) .
$$

Theorem 5.6. Suppose that assumptions (H1)-(H4) hold. Let $s$ and $\bar{r}$ be as in (C), but $s \in\left[\frac{1}{2}, \frac{3}{2}\right)$ and $\bar{r} \in\left[0, \frac{1}{2}\right)$. Then there exists $C>0$, independent of $h$, such that

$$
\left\|\partial_{t} \boldsymbol{u}_{\star}\right\|_{H^{\beta-1}\left(0, T ; \boldsymbol{W}_{\star}^{-\alpha}\right)}+\left\|\boldsymbol{u}_{\star}\right\|_{H^{\beta}\left(0, T ; \boldsymbol{W}_{\star}^{-\alpha}\right)} \leq C,
$$

for all $\alpha$ satisfying $0 \leq \alpha \leq s \leq 1+2 \alpha<2$, and for all $\beta$ satisfying $\beta<\bar{\beta}:=\frac{1+\alpha}{1+s}\left(\frac{s}{2}+\frac{1}{4}\right)$. Furthermore,

$$
\left\|\Delta_{\star} \boldsymbol{u}_{\star}\right\|_{H^{-r}\left(0, T ; \boldsymbol{W}_{\star}^{-s}\right)} \leq C
$$

for $r$ satisfying $r>\bar{r}=\frac{3}{4}-\frac{s}{2}=\frac{1}{p}-\frac{1}{2}$.

Proof. Let $\mathfrak{g}$ be the extension of $\boldsymbol{g}$ by zero off $[0, T]$. Let us define

$$
\mathfrak{u}_{\star}=\left\{\begin{array}{lll}
\mathbf{0} & \text { for } & t \in(-\infty,-1], \\
(t+1) \boldsymbol{u}_{0 \star} & \text { for } \quad t \in[-1,0], \\
\boldsymbol{u}_{\star} & \text { for } \quad t \in[0, T+1], \\
\mathbf{0} & \text { for } \quad t \in[T+1, \infty) .
\end{array}\right.
$$

Fix a cutoff function $\varphi \in \mathcal{D}(\Omega)$ which equals 1 on $[0, T]$ and vanishes on $(-1, T+1)$. Define $\tilde{\mathfrak{u}}_{\star}=\varphi \mathfrak{u}_{\star}$, and

Write

$$
\tilde{\mathfrak{g}}= \begin{cases}(1+t) \varphi^{\prime} \boldsymbol{u}_{0 \star}+\varphi \boldsymbol{u}_{0 \star}+\nu(1+t) \varphi A_{\star} \boldsymbol{u}_{0 \star}, & \text { for } t \in(-1,0), \\ \varphi \mathfrak{g}+\varphi^{\prime} \mathbf{u}_{\star}, & \text { otherwise. }\end{cases}
$$

Applying the Fourier transform, we get

$$
\partial_{t} \tilde{\mathfrak{u}}_{\star}+\nu A_{\star} \tilde{\mathfrak{u}}_{\star}=P_{\star} \tilde{\mathfrak{g}} \quad \text { in } \quad \mathcal{S}^{\prime}\left(\mathbb{R}, \boldsymbol{V}_{\star}\right) .
$$

$$
2 \pi i \xi \mathcal{F} \tilde{\mathfrak{u}}_{\star}+\nu A_{\star} \mathcal{F} \tilde{\mathfrak{u}}_{\star}=P_{\star} \mathcal{F} \tilde{\mathfrak{g}}
$$

Let $\alpha \in \mathbb{R}^{+}$. Multiply (49) by the complex conjugate of $A_{\star}^{-\alpha} \mathcal{F} \mathfrak{u}_{\star}$ and take the imaginary part to get

$$
\begin{aligned}
2 \pi|\xi|\left\|\mathcal{F} \tilde{\mathfrak{u}}_{\star}\right\|_{\boldsymbol{V}_{\star}^{-\alpha}}^{2} & \leq\|\mathcal{F} \tilde{\mathfrak{g}}\|_{\boldsymbol{W}_{\star}^{-s}}\left\|A_{\star}^{-\alpha} \mathcal{F} \tilde{\mathfrak{u}}_{\star}\right\|_{\boldsymbol{W}_{\star}^{s}} \\
& \leq\|\mathcal{F} \tilde{\mathfrak{g}}\|_{\boldsymbol{W}_{\star}^{-s}}\left\|\mathcal{F} \tilde{\mathfrak{u}}_{\star}\right\|_{\boldsymbol{V}_{\star}^{s-2 \alpha}}
\end{aligned}
$$

where we have applied (33) for $s \in[0,2)$. For $\alpha \leq s \leq 1+2 \alpha$, i.e. $-\alpha \leq s-2 \alpha \leq 1$, we interpolate to get

$$
\left\|\mathcal{F} \tilde{\mathfrak{u}}_{\star}\right\|_{\boldsymbol{V}_{\star}^{s-2 \alpha}} \leq\left\|\mathcal{F} \tilde{\mathfrak{u}}_{\star}\right\|_{V_{\star}^{-\alpha}}^{\gamma}\left\|\mathcal{F} \tilde{\mathfrak{u}}_{\star}\right\|_{\boldsymbol{V}_{\star}}^{1-\gamma}
$$

where $\gamma=\frac{2 \alpha+1-s}{1+\alpha}$. Therefore,

$$
2 \pi|\xi|\left\|\mathcal{F} \tilde{\mathfrak{u}}_{\star}\right\|_{\boldsymbol{V}_{\star}^{-\alpha}}^{2-\gamma} \leq C\|\mathcal{F} \tilde{\mathfrak{g}}\|_{\boldsymbol{W}_{\star}^{-s}}\left\|\mathcal{F} \mathfrak{u}_{\star}\right\|_{\boldsymbol{V}_{\star}}^{1-\gamma}
$$

and hence

$$
|\xi|^{\frac{2}{2-\gamma}-\mu}\left\|\mathcal{F} \tilde{\mathfrak{u}}_{\star}\right\|_{\boldsymbol{V}_{\star}^{-\alpha}}^{2} \leq C(1+|\xi|)^{-\mu}\|\mathcal{F} \tilde{\mathfrak{g}}\|_{\boldsymbol{W}_{\star}^{-s}}^{\frac{2}{2-\gamma}}\left\|\mathcal{F} \tilde{\mathfrak{u}}_{\star}\right\|_{\boldsymbol{V}_{\star}}^{\frac{2(1-\gamma)}{2-\gamma}}
$$


where $\mu=\frac{2 r}{2-\gamma}$. Integrating over $\mathbb{R}$ and using Hölder's inequality and Plancherel's equality gives

$$
\int_{\mathbb{R}}|\xi|^{\frac{2}{2-\gamma}-\mu}\left\|\mathcal{F} \tilde{\mathfrak{u}}_{\star}\right\|_{\boldsymbol{V}_{\star}^{-\alpha}}^{2} d \xi \leq C\|\tilde{\mathfrak{g}}\|_{H^{-r}\left(\mathbb{R} ; \boldsymbol{W}_{\star}^{-s}\right)}^{\frac{2}{2-\gamma}}\left\|\tilde{\mathfrak{u}}_{\star}\right\|_{L^{2}\left(\mathbb{R} ; \boldsymbol{V}_{\star}\right)}^{\frac{2(1-\gamma)}{2-\gamma}},
$$

which implies that

$$
\int_{\mathbb{R}}|\xi|^{2 \beta}\left\|\mathcal{F} \tilde{\mathfrak{u}}_{\star}\right\|_{\boldsymbol{V}_{\star}^{-\alpha}}^{2} \leq C\|\tilde{\mathfrak{g}}\|_{H^{-r}\left(0, T ; \boldsymbol{W}_{\star}^{-s}\right)}^{\frac{2}{2-\gamma}}\left\|\tilde{\mathfrak{u}}_{\star}\right\|_{\left.L^{2}\left(0, T ; \boldsymbol{V}_{\star}\right)\right)}^{\frac{2(1-\gamma)}{2-\gamma}}
$$

for $\beta<\bar{\beta}$ with $\bar{\beta}:=\frac{1+\alpha}{1+s}(1-\bar{r})$ coming from the definition of $\gamma, \mu, \bar{r}$, and $\alpha \leq s \leq 1+2 \alpha$. Next observe that we have, from (15) and (46) for $s \in\left[0, \frac{3}{2}\right)$,

$$
\|\tilde{\mathfrak{g}}\|_{H^{-r}\left(\mathbb{R} ; \boldsymbol{W}_{\star}^{-s}\right)} \leq C .
$$

Inserting (43) and (51) into (50), we arrive at

$$
\int_{\mathbb{R}}|\xi|^{2 \beta}\left\|\mathcal{F} \tilde{\mathfrak{u}}_{\star}\right\|_{V_{\star}^{-\alpha}}^{2} \leq C
$$

For $\beta \geq 0$, we write

$$
\begin{aligned}
\int_{\mathbb{R}}(1+|\xi|)^{2 \beta}\left\|\mathcal{F} \tilde{\mathfrak{u}}_{\star}\right\|_{V_{\star}^{-\alpha}}^{2} d \xi & =\int_{|\xi| \leq 1}(1+|\xi|)^{2 \beta}\left\|\mathcal{F} \tilde{\mathfrak{u}}_{\star}\right\|_{\boldsymbol{V}_{\star}^{-\alpha}}^{2} d \xi+\int_{|\xi|>1}(1+|\xi|)^{2 \beta}\left\|\mathcal{F} \mathfrak{u}_{\star}\right\|_{V_{\star}^{-\alpha}}^{2} d \xi \\
& \leq C \int_{|\xi| \leq 1}\left\|\mathcal{F} \tilde{\mathfrak{u}}_{\star}\right\|_{V_{\star}^{-\alpha}}^{2} d \xi+C \int_{|\xi|>1}|\xi|^{2 \beta}\left\|\mathcal{F} \tilde{\mathfrak{u}}_{\star}\right\|_{V_{\star}^{-\alpha}}^{2} d \xi . \\
& \leq C \int_{\mathbb{R}}\left\|\tilde{\mathfrak{u}}_{\star}\right\|_{V_{\star}}^{2} d \xi+C \int_{\mathbb{R}}|\xi|^{2 \beta}\left\|\mathcal{F} \tilde{\mathfrak{u}}_{\star}\right\|_{V_{\star}^{-\alpha}}^{2} d \xi
\end{aligned}
$$

where Plancherel's equality and the continuous embedding between $\boldsymbol{V}_{\star}$ and $\boldsymbol{V}_{\star}^{-\alpha}$ were used in the last line. The above estimate also holds trivially for $\beta<0$. Thus we get

$$
\left\|\partial_{t} \tilde{\mathfrak{u}}_{\star}\right\|_{H^{\beta-1}\left(\mathbb{R} ; V_{\star}^{-\alpha}\right)}+\left\|\tilde{\mathfrak{u}}_{\star}\right\|_{H^{\beta}\left(\mathbb{R} ; \boldsymbol{V}_{\star}^{-\alpha}\right)} \leq C .
$$

As a result of (33) for $s \in\left[0, \frac{1}{2}\right)$, we obtain

$$
\left\|\partial_{t} \tilde{\mathfrak{u}}_{\star}\right\|_{H^{\beta-1}\left(0, T ; \boldsymbol{W}_{\star}^{-\alpha}\right)}+\left\|\tilde{\mathfrak{u}}_{\star}\right\|_{H^{\beta}\left(0, T ; \boldsymbol{W}_{\star}^{-\alpha}\right)} \leq C,
$$

for all $\alpha$ satisfying $0 \leq \alpha \leq s \leq 1+2 \alpha<2$, and for all $\beta$ satisfying $\beta<\bar{\beta}:=\frac{1+\alpha}{1+s}\left(\frac{s}{2}+\frac{1}{4}\right)$. This latter inequality leads to (47).

Next, multiply (49) by $A_{\star}^{1-s} \mathcal{F} \tilde{\mathfrak{u}}_{\star}$ and take the real part to get

$$
\nu\left\|A_{\star} \mathcal{F} \tilde{\mathfrak{u}}_{\star}\right\|_{\boldsymbol{V}_{\star}^{-s}}^{2} \leq C\|\mathcal{F} \tilde{\mathfrak{g}}\|_{\boldsymbol{W}_{\star}^{-s}}\left\|A_{\star}^{1-s} \mathcal{F} \tilde{\mathfrak{u}}_{\star}\right\|_{\boldsymbol{V}_{\star}^{s}} \leq C\|\mathcal{F} \tilde{\mathfrak{g}}\|_{\boldsymbol{W}_{\star}^{-s}}\left\|A_{\star} \mathcal{F} \tilde{\mathfrak{u}}_{\star}\right\|_{\boldsymbol{V}_{\star}^{s}},
$$

where we have applied (33) for $s \in[0,2)$. Thus,

$$
(1+|\xi|)^{-2 r}\left\|A_{\star} \mathcal{F} \tilde{\mathfrak{u}}_{\star}\right\|_{\boldsymbol{V}_{\star}^{-s}}^{2} \leq C(1+|\xi|)^{-2 r}\|\mathcal{F} \tilde{\mathfrak{g}}\|_{W_{\star}^{-s}}^{2},
$$

and hence

$$
\left\|A_{\star} \tilde{\mathfrak{u}}_{\star}\right\|_{H^{-r}\left(\mathbb{R} ; \boldsymbol{V}_{\star}^{-s}\right)} \leq C\|\tilde{\mathfrak{g}}\|_{H^{-r}\left(\mathbb{R} ; \boldsymbol{W}_{\star}^{-s}\right)} .
$$

It is not hard to see from (33) that $\left\|\Delta_{\star} \boldsymbol{v}_{\star}\right\|_{\boldsymbol{W}_{\star}^{-s}} \leq C\left\|A_{\star} \boldsymbol{v}_{\star}\right\|$ for all $\boldsymbol{v}_{\star} \in \boldsymbol{V}_{\star}$ and all $s \in\left[0, \frac{3}{2}\right)$. Then, using (51) yields

$$
\left\|\Delta_{\star} \tilde{\mathfrak{u}}_{\star}\right\|_{H^{-r}\left(0, T ; \boldsymbol{W}_{\star}^{-s}\right)} \leq C,
$$

for all $r>\bar{r}$, which implies (48).

Corollary 5.7. Suppose that assumptions (H1)-(H4) hold. For $\alpha \in\left[\frac{1}{4}, \frac{1}{2}\right)$ and $\beta<\bar{\beta}=\frac{2}{5}(1+\alpha)$, it follows that

$$
\left\|\partial_{t} \boldsymbol{u}_{\star}\right\|_{H^{\beta-1}\left(0, T ; \boldsymbol{W}_{\star}^{-\alpha}\right)}+\left\|\boldsymbol{u}_{\star}\right\|_{H^{\beta}\left(0, T ; \boldsymbol{W}_{\star}^{-\alpha}\right)} \leq C,
$$

where $C>0$ is a constante independent of $h$. 
Proof. From $s \in\left[\frac{1}{2}, \frac{3}{2}\right)$ and $0 \leq \alpha \leq s \leq 1+2 \alpha<2$, we obtain $\alpha \in\left[\frac{1}{4}, \frac{1}{2}\right)$. Next note that $\frac{1}{1+s}\left(\frac{s}{2}+\frac{1}{4}\right)$ reaches its maximum $\frac{2}{5}$ at $s=\frac{3}{2}$. Therefore we can simplify the expression $\bar{\beta}$ in term of $\alpha$ only as $\bar{\beta}=\frac{2}{5}(1+\alpha)$ in $(47)$.

Using (23), one can also prove the following.

Corollary 5.8. Assume that assumptions (H1)-(H4) hold. Then, for $\alpha \in\left[\frac{1}{4}, \frac{1}{2}\right)$ and $\beta<\bar{\beta}=\frac{2}{5}(1+\alpha)$, it follows that there exists a constant $C>0$, independent of $h$, such that

$$
\left\|\partial_{t} \boldsymbol{u}_{h}\right\|_{H^{\beta-1}\left(0, T ; \tilde{\boldsymbol{H}}_{0}^{-\alpha}(\Omega)\right)}+\left\|\boldsymbol{u}_{h}\right\|_{H^{\beta}\left(0, T ; \tilde{\boldsymbol{H}}_{0}^{-\alpha}(\Omega)\right)} \leq C .
$$

Furthermore, for $s \in\left[\frac{1}{2}, \frac{3}{2}\right]$ and $r$ such that $r>\bar{r}=\frac{3}{4}-\frac{s}{2}=\frac{1}{p}-\frac{1}{2}$, it follows that

$$
\left\|\Delta_{h} \boldsymbol{u}_{h}\right\|_{H^{-r}\left(0, T ; \tilde{\boldsymbol{H}}_{0}^{-s}(\Omega)\right)} \leq C .
$$

We now proceed to obtain an estimate for $p_{h}$.

Lemma 5.9. Suppose that conditions (H1)-(H4) hold. There exists a constant $C>0$, independent of $h$, such that, for $s \in\left[\frac{1}{2}, \frac{7}{10}\right]$ and $r>\bar{r}=\frac{3}{4}-\frac{s}{2}$,

$$
\left\|p_{h}\right\|_{H^{-r}\left(0, T ; H^{1-s}(\Omega)\right)} \leq C,
$$

where $C>0$ is a constant independent of $h$.

Proof. First we write (52) as

$$
\left\|\partial_{t} \boldsymbol{u}_{\star}\right\|_{H^{-r}\left(0, T ; \boldsymbol{W}_{\star}^{-\alpha}\right)} \leq C,
$$

where $\alpha \in\left[\frac{1}{4}, \frac{1}{2}\right)$ and $r>\tilde{r}:=1-\bar{\beta}=\frac{3}{5}-\frac{2}{5} \alpha$. As a result, we have that

$$
\left\|\partial_{t} \boldsymbol{u}_{\star}\right\|_{H^{-r}\left(0, T ; \boldsymbol{W}_{\star}^{-s}\right)} \leq C
$$

holds for $\alpha \leq s$ and $\tilde{r} \leq \bar{r}$ provided that $s \in\left[\frac{1}{2}, \frac{7}{10}\right]$ and $r>\bar{r}=\frac{3}{4}-\frac{s}{2}$.

From (45), we bound

$$
\begin{aligned}
\left\|p_{h}\right\|_{H^{1-s}(\Omega)} & \leq \sup _{\boldsymbol{v}_{\star} \in \boldsymbol{W}_{\star} \backslash\{0\}} \frac{\left(\nabla p_{h}, \boldsymbol{v}_{\star}\right)}{\left\|\boldsymbol{v}_{\star}\right\| \boldsymbol{W}_{\star}^{s}} \\
& \leq C\left(\left\|\partial_{t} \boldsymbol{u}_{\star}\right\|_{\boldsymbol{W}_{\star}^{-s}}+\left\|\Delta_{\star} \boldsymbol{u}_{\star}\right\|_{\boldsymbol{W}_{\star}^{-s}}+\left\|\mathcal{N}_{\star}\left(\boldsymbol{u}_{\star}, \boldsymbol{u}_{\star}\right)\right\|_{\boldsymbol{W}_{\star}^{-s}}+\left\|\boldsymbol{f}_{h}\right\|_{\boldsymbol{W}_{\star}^{-s}}\right) \\
& \leq C\left(\left\|\partial_{t} \boldsymbol{u}_{\star}\right\|_{\boldsymbol{W}_{\star}^{-\alpha}}+\left\|\Delta_{\star} \boldsymbol{u}_{\star}\right\|_{\boldsymbol{W}_{\star}^{-s}}+\left\|\mathcal{N}_{\star}\left(\boldsymbol{u}_{\star}, \boldsymbol{u}_{\star}\right)\right\|_{\boldsymbol{W}_{\star}^{-s}}+\left\|\boldsymbol{f}_{h}\right\|_{\tilde{H}_{0}^{-s}(\Omega)}\right) .
\end{aligned}
$$

The proof is completed via (56), (48), (46) and (16).

\section{Convergence towards WeAK And Suitable WeAK SOlutions}

In this section we will prove that the sequence of the approximate solutions provided by scheme (17) converges towards a weak solution in the sense of Definition 2.3 and towards a suitable weak solution in the sense of Definition 2.4. In order for these convergence results to hold, we will need to use the following compactness results à la Aubin-Lions.

The following compactness result is due to Lions [32].

Lemma 6.1. Let $H_{0} \hookrightarrow H \hookrightarrow H_{1}$ be three Hilbert spaces with dense and continuous embedding. Assume that the embedding $H_{0} \hookrightarrow H$ is compact. Then $L^{2}\left(0, T ; H_{0}\right) \cap H^{\gamma}\left(0, T ; H_{1}\right)$ embeds compactly in $L^{2}(0, T ; H)$ for $\gamma>0$.

The proof of the two following compactness result can be found in [22, Ap. A.1, A.2].

Lemma 6.2. Let $X \hookrightarrow Y$ be two Hilbert spaces with compact embedding. Then $H^{\beta}(0, T ; X)$ embeds continuously and compactly in $C^{0}([0, T] ; Y)$ for $\beta>\frac{1}{2}$.

Lemma 6.3. Let $H_{0} \hookrightarrow H_{1}$ be two Hilbert spaces with compact embedding. Let $\gamma>0$ and $\gamma>\mu$, then the injection $H^{\gamma}\left(0, T ; H_{0}\right) \hookrightarrow H^{\mu}\left(0, T H_{1}\right)$ is compact. 
Theorem 6.4. Assume that hypotheses (H1)-(H4) are satisfied. Then there exists a subsequence (denoted in the same way) of approximate solutions $\left(\boldsymbol{u}_{h}, p_{h}\right)$ converging toward a weak solution given in Definition (2.3) in the following sense as $h \rightarrow 0$ :

$$
\boldsymbol{u}_{h} \rightarrow \boldsymbol{u} \text { in } L^{2}\left(0, T ; \boldsymbol{H}_{0}^{1}(\Omega)\right) \text { - weak and in } L^{2}\left(0, T ; \boldsymbol{H}^{\beta}(\Omega)\right)-\text { strong for all } \beta<1
$$

and

$$
p_{h} \rightarrow p \text { in } H^{-r}\left(0, T ; H^{\delta}(\Omega)\right)-\text { weak for all } \delta \in\left[\frac{3}{10}, \frac{1}{2}\right] \text { and } r>\frac{1}{4}+\frac{\delta}{2} \text {. }
$$

Proof. Let $\boldsymbol{v} \in H^{r}\left(0, T ; \tilde{\boldsymbol{H}}_{0}^{s}(\Omega)\right)$, for $s \in\left(\frac{1}{2}, \frac{7}{10}\right]$ and $r>\frac{3}{4}-\frac{s}{2}$, and $q \in L^{2}\left(0, T ; H_{\int=0}^{1}(\Omega)\right)$. From (11) and (12), we are allowed to construct three sequences $\left\{\boldsymbol{v}_{h}\right\}_{h>0} \subset H^{r}\left(0, T ; \boldsymbol{W}_{h}\right),\left\{\tilde{\boldsymbol{v}}_{h}\right\}_{h>0} \subset$ $H^{r}\left(0, T ; \tilde{\boldsymbol{W}}_{h}\right)$ and $\left\{q_{h}\right\}_{h>0} \subset L^{2}\left(0, T ; Q_{h}\right)$ such that $\boldsymbol{v}_{h} \rightarrow \boldsymbol{v}$ in $H^{r}\left(0, T ; \tilde{\boldsymbol{H}}_{0}^{s}(\Omega)\right)$-strong, $\tilde{\boldsymbol{v}}_{h} \rightarrow \mathbf{0}$ in $L^{2}\left(0, T ; \boldsymbol{L}^{2}(\Omega)\right)$-strong and $q_{h} \rightarrow q$ in $L^{2}\left(0, T ; H_{\int=0}^{1}(\Omega)\right)$-strong as $h \rightarrow 0$.

By virtue of (43), (53), (54) and (55), we know that there exist a subsequence of $\left\{\boldsymbol{v}_{h}\right\}_{h>0}$ and $\left\{p_{h}\right\}_{h>0}$, still denoted by itself, and a pair $(\boldsymbol{u}, p)$ such that

$$
\begin{aligned}
\boldsymbol{u}_{h} & \rightarrow \boldsymbol{u} \text { in } L^{\infty}\left(0, T ; \boldsymbol{L}^{2}(\Omega)\right)-\text { weak- }, \\
\boldsymbol{u}_{h} & \rightarrow \boldsymbol{u} \text { in } L^{2}\left(0, T ; \boldsymbol{H}_{0}^{1}(\Omega)\right)-\text { weak, } \\
\partial_{t} \boldsymbol{u}_{h} & \rightarrow \partial_{t} \boldsymbol{u} \text { in } H^{-r}\left(0, T ; \tilde{\boldsymbol{H}}_{0}^{-s}(\Omega)\right) \text { - weak, } \\
\Delta_{h} \boldsymbol{u}_{h} & \rightarrow \Delta \boldsymbol{u} \text { in } H^{-r}\left(0, T ; \tilde{\boldsymbol{H}}_{0}^{-s}(\Omega)\right) \text { - weak, }
\end{aligned}
$$

and

$$
\nabla p_{h} \rightarrow \nabla p \text { in } H^{-r}\left(0, T ; \tilde{\boldsymbol{H}}_{0}^{-s}(\Omega)\right) \text { - weak, }
$$

for all $s \in\left(\frac{1}{2}, \frac{7}{10}\right]$ and $r>\frac{3}{4}-\frac{s}{2}$. Observe that we have used that the fact that $\tilde{\boldsymbol{H}}^{-s}(\Omega)$ coincides with $\boldsymbol{H}_{0}^{-s}(\Omega)$ for $s \in\left(\frac{1}{4}, \frac{7}{10}\right]$ for the pressure. We also have, from (43), that

$$
\tilde{\boldsymbol{u}}_{h} \rightarrow 0 \quad \text { in } \quad L^{2}\left(0, T ; \boldsymbol{L}^{2}(\Omega)\right)-\text { strong, }
$$

since

$$
\frac{\nu^{\frac{1}{2}}}{h}\left\|\tilde{\boldsymbol{u}}_{h}\right\|_{L^{2}\left(0, T ; \boldsymbol{L}^{2}(\Omega)\right)} \leq\left\|\tau^{-\frac{1}{2}} \tilde{\boldsymbol{u}}_{h}\right\|_{L^{2}\left(0, T ; \boldsymbol{L}^{2}(\Omega)\right)} \leq C .
$$

We can pass to the limit in (17b). Thus we find that $\nabla \cdot \boldsymbol{u}=0$ in $(0, T) \times \Omega$, whence $\boldsymbol{u} \in$ $L^{\infty}(0, T ; \boldsymbol{H}) \cap L^{2}(0, T ; \boldsymbol{V})$. For the trilinear terms, we proceed as follows. By Lemma 6.1 , we have that

$$
\boldsymbol{u}_{h} \rightarrow \boldsymbol{u} \text { in } L^{2}\left(0, T ; \boldsymbol{H}^{\beta}(\Omega)\right) \text { - strong for all } \beta<1,
$$

since $\left\{\boldsymbol{u}_{h}\right\}_{h>0}$ is bounded in $L^{2}\left(0, T ; \boldsymbol{H}_{0}^{1}(\Omega)\right) \cap H^{\beta}\left((0, T) ; \tilde{\boldsymbol{H}}_{0}^{-\alpha}(\Omega)\right)$ for $\alpha \in\left[\frac{1}{4}, \frac{1}{2}\right)$ and $0<\beta<\frac{2}{5}(1+\alpha)$ from (43) and (53). Therefore,

$$
\mathcal{N}\left(\boldsymbol{u}_{h}, \boldsymbol{u}_{h}\right) \rightarrow \mathcal{N}(\boldsymbol{u}, \boldsymbol{u}) \text { in } \mathcal{D}^{\prime}((0, T) \times \Omega) .
$$

As a consequence of (46), we obtain

$$
\mathcal{N}\left(\boldsymbol{u}_{h}, \boldsymbol{u}_{h}\right) \rightarrow \mathcal{N}(\boldsymbol{u}, \boldsymbol{u}) \text { in } H^{-r}\left(0, T ; \tilde{\boldsymbol{H}}_{0}^{-s}(\Omega)\right) .
$$

On passing to the limit in (17b), we have had that $\nabla \cdot \boldsymbol{u}=0$ in $(0, T) \times \Omega$, thereby

$$
\mathcal{N}\left(\boldsymbol{u}_{h}, \boldsymbol{u}_{h}\right) \rightarrow(\boldsymbol{u} \cdot \nabla) \boldsymbol{u} \text { in } H^{-r}\left(0, T ; \tilde{\boldsymbol{H}}_{0}^{-s}(\Omega)\right) .
$$

By an analogous argument, we find that

$$
\tilde{\mathcal{N}}\left(\boldsymbol{u}_{h}, \tilde{\boldsymbol{u}}_{h}\right) \rightarrow \mathbf{0} \text { in } H^{-r}\left(0, T ; \tilde{\boldsymbol{H}}_{0}^{-s}(\Omega)\right),
$$

where $\left\langle\tilde{\mathcal{N}}\left(\boldsymbol{u}_{h}, \tilde{\boldsymbol{w}}_{h}\right), \boldsymbol{v}_{h}\right\rangle=b\left(\boldsymbol{u}_{h}, \boldsymbol{v}_{h}, \tilde{\boldsymbol{w}}_{h}\right)$ for all $\boldsymbol{u}_{h}, \boldsymbol{w}_{h} \in \boldsymbol{W}_{h}$ and $\tilde{\boldsymbol{v}}_{h} \in \tilde{\boldsymbol{W}}_{h}$.

From the above convergences, it is easy to see that

$$
\partial_{t} \boldsymbol{u}_{h}+\mathcal{N}\left(\boldsymbol{u}_{h}, \boldsymbol{u}_{h}\right)-\nu \Delta_{h} \boldsymbol{u}_{h}+\nabla p_{h}-\tilde{\mathcal{N}}\left(\boldsymbol{u}_{h}, \tilde{\boldsymbol{u}}_{h}\right)-\boldsymbol{f}_{h} \rightarrow \partial_{t} \boldsymbol{u}+\left(\boldsymbol{u}_{h} \cdot \nabla\right) \boldsymbol{u}_{h}-\nu \Delta \boldsymbol{u}+\nabla p-\boldsymbol{f} .
$$

in $H^{-r}\left(0, T ; \tilde{\boldsymbol{H}}_{0}^{-s}(\Omega)\right)$ as $h \rightarrow 0$. 
For the initial condition, we have that $\boldsymbol{u}_{h} \rightarrow \boldsymbol{u}$ in $C^{0}\left([0, T] ; \tilde{\boldsymbol{H}}_{0}^{-\alpha}(\Omega)\right)$-strong for $\alpha \in\left(\frac{1}{4}, \frac{1}{2}\right)$ by Lemma 6.2; therefore, $\boldsymbol{u}_{h}(0) \rightarrow \boldsymbol{u}(0)$ in $\tilde{\boldsymbol{H}}_{0}^{-\alpha}(\Omega)$. Furthermore, it follows from (18) and (27) that $\boldsymbol{u}_{0 h} \rightarrow \boldsymbol{u}_{0}$ in $\tilde{\boldsymbol{H}}_{0}^{-\alpha}(\Omega)$. We have thus shown that $\boldsymbol{u}(0)=\boldsymbol{u}_{0}$.

The energy inequality can be verified by the lower semicontinuity of the norm for the weak topology; for complete details, see [4].

Theorem 6.5. Under hypotheses (H1)-(H4), the sequence of approximate solutions $\left(\boldsymbol{u}_{h}, p_{h}\right)$ converges, up to a subsequence, to a suitable weak solution given in Definition 2.4 as $h \rightarrow 0$.

Proof. Let $\phi \in \mathcal{D}\left((0, T) \times \Omega ; \mathbb{R}^{+}\right)$and substitute $\boldsymbol{v}_{h}=\pi_{\boldsymbol{W}_{h}}\left(\boldsymbol{u}_{h} \phi\right)$ into (17a) to get

$$
\begin{aligned}
& \int_{0}^{T}\left\{\left(\partial_{t} \boldsymbol{u}_{h}, \pi_{\boldsymbol{W}_{h}}\left(\boldsymbol{u}_{h} \phi\right)\right)+b\left(\boldsymbol{u}_{h}, \boldsymbol{u}_{h}, \pi_{\boldsymbol{W}_{h}}\left(\boldsymbol{u}_{h} \phi\right)\right)+\nu\left(\nabla \boldsymbol{u}_{h}, \nabla \pi_{\boldsymbol{W}_{h}}\left(\boldsymbol{u}_{h} \phi\right)\right)\right. \\
& \left.-\left(p_{h}, \nabla \cdot \pi_{\boldsymbol{W}_{h}}\left(\boldsymbol{u}_{h} \phi\right)\right)-b\left(\boldsymbol{u}_{h}, \pi_{\boldsymbol{W}_{h}}\left(\boldsymbol{u}_{h} \phi\right), \tilde{\boldsymbol{u}}_{h}\right)-\left(\boldsymbol{f}_{h}, \pi_{\boldsymbol{W}_{h}}\left(\boldsymbol{u}_{h} \phi\right)\right)\right\} \mathrm{d} t=0 .
\end{aligned}
$$

We are ready to take the limit in (60) as $h \rightarrow 0$ so as to prove that the weak solution $(\boldsymbol{u}, p)$ found in Theorem 6.4 is suitable. We will only focus on passing to the limit in the terms of (60) involving the subscale velocity $\tilde{\boldsymbol{u}}_{h}$ and the pressure term. The remaining terms appear in a rudimentary finite element formulation so that a proof can be found in [24]. In particular, from (13) and (9) and in virtue of Lemma 6.3, it follows that

$$
\begin{gathered}
\lim _{h \rightarrow 0} \int_{0}^{T}\left(\partial_{t} \boldsymbol{u}_{h}, \boldsymbol{u}_{h} \phi\right) \mathrm{d} t=-\frac{1}{2} \int_{0}^{T}\left(|\boldsymbol{u}|^{2}, \partial_{t} \phi\right) \mathrm{d} t \\
\lim _{h \rightarrow 0} \int_{0}^{T} b\left(\boldsymbol{u}_{h}, \boldsymbol{u}_{h}, \pi_{\boldsymbol{W}_{h}}\left(\boldsymbol{u}_{h} \phi\right)\right) \mathrm{d} t=-\frac{1}{2} \int_{0}^{T}\left(|\boldsymbol{u}|^{2} \boldsymbol{u}, \nabla \phi\right) d t, \\
\liminf _{h \rightarrow 0} \int_{0}^{T} \nu\left(\nabla \boldsymbol{u}_{h}, \nabla \pi_{W_{h}}\left(\boldsymbol{u}_{h} \phi\right)\right) \mathrm{d} t \geq \int_{0}^{T}\left(|\nabla \boldsymbol{u}|^{2}, \phi\right) \mathrm{d} t-\int_{0}^{T}\left(\frac{1}{2}|\boldsymbol{u}|^{2}, \Delta \phi\right) \mathrm{d} t,
\end{gathered}
$$

and

$$
\lim _{h \rightarrow 0}-\int_{0}^{T}\left\langle\boldsymbol{f}_{h}, \boldsymbol{u}_{h} \phi\right\rangle \mathrm{d} t=-\int_{0}^{T}\langle\boldsymbol{f}, \boldsymbol{u} \phi\rangle \mathrm{d} t .
$$

To begin with, we first turn our attention to passing to the limit in the convective term.

$$
\begin{aligned}
b\left(\boldsymbol{u}_{h}, \pi_{W_{h}}\left(\boldsymbol{u}_{h} \phi\right), \tilde{\boldsymbol{u}}_{h}\right) \mathrm{d} t= & b\left(\boldsymbol{u}_{h}, \boldsymbol{u}_{h} \phi, \tilde{\boldsymbol{u}}_{h}\right) \mathrm{d} t+b\left(\boldsymbol{u}_{h}, \pi_{\boldsymbol{W}_{h}}\left(\boldsymbol{u}_{h} \phi\right)-\boldsymbol{u}_{h} \phi, \tilde{\boldsymbol{u}}_{h}\right) \mathrm{d} t \\
= & b\left(\boldsymbol{u}_{h}, \boldsymbol{u}_{h}, \tilde{\boldsymbol{u}}_{h} \phi\right)+\left(\boldsymbol{u}_{h} \cdot \nabla \phi \boldsymbol{u}_{h}, \tilde{\boldsymbol{u}}_{h}\right)+b\left(\boldsymbol{u}_{h}, \pi_{\boldsymbol{W}_{h}}^{\perp}\left(\boldsymbol{u}_{h} \phi\right), \tilde{\boldsymbol{u}}_{h}\right) \\
= & \left(\pi_{\boldsymbol{W}_{h}}^{\perp}\left(\mathcal{N}\left(\boldsymbol{u}_{h}, \boldsymbol{u}_{h}\right) \phi\right), \tilde{\boldsymbol{u}}_{h}\right)+\left(\pi_{\boldsymbol{W}_{h}}^{\perp}\left(\boldsymbol{u}_{h} \cdot \nabla \phi \boldsymbol{u}_{h}\right), \tilde{\boldsymbol{u}}_{h}\right) \\
& +b\left(\boldsymbol{u}_{h}, \pi_{\boldsymbol{W}_{h}}^{\perp}\left(\boldsymbol{u}_{h} \phi\right), \tilde{\boldsymbol{u}}_{h}\right) .
\end{aligned}
$$

From (13) and (6), we have:

$$
\begin{aligned}
\int_{0}^{T}\left(\pi_{\boldsymbol{W}_{h}}^{\perp}\left(\mathcal{N}\left(\boldsymbol{u}_{h}, \boldsymbol{u}_{h}\right) \phi\right), \tilde{\boldsymbol{u}}_{h}\right) \mathrm{d} t & \left.\leq \int_{0}^{T} \| \pi_{\boldsymbol{W}_{h}}^{\perp}\left(\mathcal{N}\left(\boldsymbol{u}_{h}, \boldsymbol{u}_{h}\right) \phi\right), \tilde{\boldsymbol{u}}_{h}\right)\|\| \tilde{\boldsymbol{u}}_{h}\left\|\mathrm{~d} t \leq C \int_{0}^{T} h\right\| \boldsymbol{u}_{h}\left\|_{\boldsymbol{L}^{\infty}(\Omega)}\right\| \nabla \boldsymbol{u}_{h}\|\| \tilde{\boldsymbol{u}}_{h} \| \mathrm{d} t \\
& \leq C\left(\int_{0}^{T} \tau h^{2}\left\|\boldsymbol{u}_{h}\right\|_{\boldsymbol{L}^{\infty}(\Omega)}^{2}\left\|\nabla \boldsymbol{u}_{h}\right\|^{2} \mathrm{~d} t\right)^{\frac{1}{2}}\left(\int_{0}^{T} \tau^{-1}\left\|\tilde{\boldsymbol{u}}_{h}\right\|^{2} \mathrm{~d} t\right)^{\frac{1}{2}} \\
& \leq C h^{\frac{3}{4}}\left\|\boldsymbol{u}_{h}\right\|_{L^{\infty}\left(0, T ; L^{2}(\Omega)\right)}^{\frac{1}{2}}\left\|\boldsymbol{u}_{h}\right\|_{L^{2}\left(0, T ; \boldsymbol{H}_{0}^{1}(\Omega)\right)}\left\|\tilde{\boldsymbol{u}}_{h}\right\|_{\tau^{-\frac{1}{2}} L^{2}\left(0, T ; \boldsymbol{L}^{2}(\Omega)\right)}
\end{aligned}
$$

and hence

$$
\lim _{h \rightarrow 0} \int_{0}^{T}\left(\pi_{\boldsymbol{W}_{h}}^{\perp}\left(\mathcal{N}\left(\boldsymbol{u}_{h}, \boldsymbol{u}_{h}\right) \phi\right), \tilde{\boldsymbol{u}}_{h}\right) \mathrm{d} t=0
$$

Analogously, we bound

$$
\int_{0}^{T}\left(\boldsymbol{u}_{h} \cdot \nabla \phi, \boldsymbol{u}_{h} \cdot \tilde{\boldsymbol{u}}_{h}\right) \mathrm{d} t \leq C T h^{\frac{3}{4}}\left\|\boldsymbol{u}_{h}\right\|_{L^{\infty}\left(0, T ; \boldsymbol{L}^{2}(\Omega)\right)}\left\|\tilde{\boldsymbol{u}}_{h}\right\|_{\tau^{-\frac{1}{2}} L\left(0, T ; \boldsymbol{L}^{2}(\Omega)\right)}
$$


and

$$
\int_{0}^{T} b\left(\boldsymbol{u}_{h}, \pi_{\boldsymbol{W}_{h}}\left(\boldsymbol{u}_{h} \phi\right)-\boldsymbol{u}_{h} \phi, \tilde{\boldsymbol{u}}_{h}\right) \mathrm{d} t \leq C h^{\frac{3}{4}}\left\|\boldsymbol{u}_{h}\right\|_{L^{\infty}\left(0, T ; L^{2}(\Omega)\right)}^{\frac{1}{2}}\left\|\boldsymbol{u}_{h}\right\|_{L^{2}\left(0, T ; \boldsymbol{H}_{0}^{1}(\Omega)\right)}\left\|\tilde{\boldsymbol{u}}_{h}\right\|_{\tau^{-\frac{1}{2}} L^{2}\left(0, T ; L^{2}(\Omega)\right)} .
$$

Thus

$$
\lim _{h \rightarrow 0} \int_{0}^{T}\left(\boldsymbol{u}_{h} \cdot \nabla \phi, \boldsymbol{u}_{h} \cdot \tilde{\boldsymbol{u}}_{h}\right) \mathrm{d} t=0
$$

and

$$
\lim _{h \rightarrow 0} \int_{0}^{T} b\left(\boldsymbol{u}_{h}, \pi_{W_{h}}\left(\boldsymbol{u}_{h} \phi\right)-\boldsymbol{u}_{h} \phi, \tilde{\boldsymbol{u}}_{h}\right) d t=0 .
$$

For the "viscous" term, it is not hard to see that

$$
\liminf _{h \rightarrow 0} \int_{0}^{T} \tau^{-1}\left(\left|\tilde{\boldsymbol{u}}_{h}\right|^{2}, \phi\right) \mathrm{d} t \geq 0 .
$$

For the pressure terms, we write

$$
\int_{0}^{T}\left(p_{h}, \nabla \cdot \pi_{\boldsymbol{W}_{h}}\left(\boldsymbol{u}_{h} \phi\right)\right) \mathrm{d} t=\int_{0}^{T}\left(p_{h} \boldsymbol{u}_{h}, \nabla \phi\right) d t+\int_{0}^{T}\left(p_{h}, \nabla \cdot\left(\pi_{\boldsymbol{W}_{h}}\left(\boldsymbol{u}_{h} \phi\right)-\left(\boldsymbol{u}_{h} \phi\right)\right)\right) \mathrm{d} t+\int_{0}^{T}\left(\phi p_{h}, \nabla \cdot \boldsymbol{u}_{h}\right) \mathrm{d} t .
$$

It was proved in [22] that

$$
\lim _{h \rightarrow 0} \int_{0}^{T}\left(p_{h} \boldsymbol{u}_{h}, \nabla \phi\right) \mathrm{d} t=\int_{0}^{T}(p u, \nabla \phi) \mathrm{d} t
$$

and

$$
\lim _{h \rightarrow 0}=\int_{0}^{T}\left(p_{h}, \nabla \cdot\left(\pi_{\boldsymbol{W}_{h}}\left(\boldsymbol{u}_{h} \phi\right)-\left(\boldsymbol{u}_{h} \phi\right)\right)\right) \mathrm{d} t=0 .
$$

For the remaining pressure terms, we use (17b) with $q_{h}=\pi_{Q_{h}}\left(\phi p_{h}\right)$ to obtain

$$
\begin{aligned}
\int_{0}^{T}\left(\phi p_{h}, \nabla \cdot \boldsymbol{u}_{h}\right) \mathrm{d} t & +\int_{0}^{T}\left(\nabla p_{h}, \tilde{\boldsymbol{u}}_{h} \phi\right)=\int_{0}^{T}\left(p_{h} \phi-\pi_{Q_{h}}\left(p_{h} \phi\right), \nabla \cdot \boldsymbol{u}_{h}\right) \mathrm{d} t \\
& +\int_{0}^{T}\left(\nabla\left(p_{h} \phi\right)-\nabla \pi_{Q_{h}}\left(p_{h} \phi\right), \tilde{\boldsymbol{u}}_{h}\right) \mathrm{d} t-\int_{0}^{T}\left(p_{h} \nabla \phi, \tilde{\boldsymbol{u}}_{h}\right) \mathrm{d} t .
\end{aligned}
$$

We know from [22] that

$$
\lim _{h \rightarrow 0} \int_{0}^{T}\left(p_{h} \phi-\pi_{Q_{h}}\left(p_{h} \phi\right), \nabla \cdot \boldsymbol{u}_{h}\right) \mathrm{d} t=0 .
$$

Let $\varepsilon>0$ and set $s=\frac{1}{2}+\frac{16}{9} \varepsilon$ and $\bar{r}=\frac{3}{4}-\frac{s}{2}=\frac{1}{4}-\frac{4}{9} \varepsilon$. Now choose $r=\frac{1}{2}-\frac{4}{9} \varepsilon$. Moreover, set $\alpha=\frac{1}{4}-\frac{5}{9} \varepsilon$ and $\bar{\beta}=\frac{2}{5}(1+\alpha)=\frac{1}{5}-\frac{2}{9} \varepsilon$. Thus we have $1-s>\alpha$ and $\bar{\beta}>r$ since

$$
1-s=\frac{1}{2}-\frac{16}{9} \varepsilon>\frac{1}{2}\left(\frac{1}{2}-\frac{16}{9} \varepsilon\right)=\frac{1}{4}-\frac{4}{9} \varepsilon>\frac{1}{4}-\frac{5}{9} \varepsilon=\alpha
$$

and

$$
r=\frac{1}{2}-\frac{4}{9} \varepsilon<\frac{1}{2}-\frac{2}{9} \varepsilon=\frac{2}{5}\left(\frac{5}{4}-\frac{5}{9} \varepsilon\right)=\frac{2}{5}\left(1+\frac{1}{4}-\frac{5}{9} \varepsilon\right)=\frac{2}{5}(1+\alpha)=\bar{\beta} .
$$

From the a priori energy estimates (52) and (55) and the commutator property (14), our choice of parameters yields

$$
\begin{aligned}
\int_{0}^{T}\left(\nabla(p \phi)-\nabla \pi_{Q_{h}}\left(p_{h} \phi\right), \tilde{\boldsymbol{u}}_{h}\right) \mathrm{d} t & \leq\left\|\nabla(p \phi)-\nabla \pi_{Q_{h}}\left(p_{h} \phi\right)\right\|_{H^{-r}\left(0, T ; \boldsymbol{L}^{2}(\Omega)\right)}\left\|\tilde{\boldsymbol{u}}_{h}\right\|_{H^{r}\left(0, T ; \boldsymbol{L}^{2}(\Omega)\right)} \\
& \leq C h^{1-s-\alpha}\left\|p_{h}\right\|_{H^{-r}\left(0, T ; H^{1-s}(\Omega)\right)}\left\|\tilde{\boldsymbol{u}}_{h}\right\|_{h^{\alpha} H^{r}\left(0, T ; \boldsymbol{L}^{2}(\Omega)\right)}
\end{aligned}
$$

and hence

$$
\lim _{h \rightarrow 0} \int_{0}^{T}\left(\nabla(\phi)-\nabla \pi_{Q_{h}}\left(p_{h} \phi\right), \tilde{\boldsymbol{u}}_{h}\right) \mathrm{d} t=0 .
$$


Finally, it is easy to see in a similar fashion that

$$
\lim _{h \rightarrow 0} \int_{0}^{T}\left(\phi p_{h}, \nabla \cdot \boldsymbol{u}_{h}\right) \mathrm{d} t=\lim _{h \rightarrow} \int_{0}^{T}\left(\phi p_{h}-\pi_{\boldsymbol{W}_{h}}\left(\phi p_{h}\right), \nabla \cdot \boldsymbol{u}_{h}\right) \mathrm{d} t=0 .
$$

\section{Appendix A. Proof of the inverse inequalities (8)}

To prove inequalities (8), we follow very closely the arguments developed in [8, Thm. 4.5.11].

We first need to introduce an equivalent norm for fractional order Hilbert spaces as follows. Let $s \in(0,1)$. Then

where

$$
\|u\|_{H^{s}(\Omega)}^{2}=\|u\|^{2}+|u|_{H^{s}(\Omega)}^{2},
$$

$$
|u|_{H^{s}(\Omega)}^{2}=\int_{\Omega} \int_{\Omega} \frac{|u(\boldsymbol{x})-u(\boldsymbol{y})|^{2}}{|\boldsymbol{x}-\boldsymbol{y}|^{3+2 s}} \mathrm{~d} \boldsymbol{x} \mathrm{d} \boldsymbol{y} .
$$

Given $(K, \mathcal{P}, \Sigma)$, we define $(\tilde{K}, \tilde{P}, \tilde{\Sigma})$ where $\hat{K}=\left\{\left(1 / h_{K}\right) \boldsymbol{x}: \boldsymbol{x} \in K\right\}$. Thus, if $u_{h}$ is a function defined on $K$, then $\hat{u}_{h}$ is defined on $\tilde{K}$ by

$$
\hat{u}(\hat{\boldsymbol{x}})=u\left(h_{K}^{-1} \boldsymbol{x}\right) \quad \text { for all } \quad \hat{\boldsymbol{x}} \in \hat{K} .
$$

Thus we can write

$$
\left\|\nabla u_{h}\right\|_{\boldsymbol{L}^{2}(K)}=h_{K}^{\frac{1}{2}}\left\|\hat{\nabla} \hat{u}_{h}\right\|_{\boldsymbol{L}^{2}(K)} .
$$

As $\hat{\nabla} \hat{u}_{h}$ belongs to a space of finite and fixed dimension on $\hat{K}$, on which all norms are equivalent, it is not hard to see that there is a constant $C_{\hat{T}}>0$ such that

$$
\left\|\hat{\nabla} \hat{u}_{h}\right\|_{\boldsymbol{L}^{2}(K)} \leq C_{\hat{T}}\left|\hat{u}_{h}\right|_{H^{s}(\hat{K})} .
$$

Reverting to $K$, this leads to

and hence

$$
\|\hat{\nabla} \hat{u}\|_{\boldsymbol{L}^{2}} \leq C_{\hat{T}} h_{K}^{-\frac{3}{2}+s}\left|u_{h}\right|_{H^{s}(K)}
$$

$$
\left\|\nabla u_{h}\right\|_{\boldsymbol{L}^{2}(K)} \leq C_{\hat{T}} h_{K}^{-1+s}\left\|u_{h}\right\|_{H^{s}(\Omega)} .
$$

An argument in the proof of [8, Prop. 4.4.11] shows that if $(\tilde{K}, \tilde{\mathcal{P}}, \tilde{\Sigma})$ is a referent element, we have that there exists a constant $C_{\tilde{T}}>0$ such that $C_{\hat{T}} \leq C_{\tilde{T}}$. Summing over all elements $K$ and using the quasi-uniformity of the mesh leads to

$$
\left\|\nabla u_{h}\right\| \leq C h^{-1+s}\left(\sum_{K \in \mathcal{T}_{h}}\left\|u_{h}\right\|_{H^{s}(\Omega)}^{2}\right) .
$$

Then (8) follows because the sum of the fractional norms over all elements is smaller than the fractional norm over the union of the elements.

\section{REFERENCES}

[1] Adams, R. A.; Fournier, J. J. F. Sobolev spaces, volume 140 of Pure and Applied Mathematics (Amsterdam). Elsevier/Academic Press, Amsterdam, second edition, 2003.

[2] BADiA, S. On stabilized finite element methods based on the Scott-Zhang projector. Circumventing the inf-sup condition for the Stokes problem. Computer Methods in Applied Mechanics and Engineering, 247-248(0):65-72, 2012.

[3] Badia, S.; Codina, R.; Gutiérrez-Santacreu, J. V. Long-term stability estimates and existence of a global attractor in a finite element approximation of the Navier-Stokes equations with numerical subgrid scale modeling. SIAM J. Numer. Anal., 48(3):1013-1037, 2010.

[4] Badia, S; Gutiérrez-Santacreu, J. V. Convergence towards weak solutions of the Navier-Stokes equations for a finite element approximation with numerical subgrid-scale modelling. IMA J. Numer. Anal., 34(3):1193-1221, 2014.

[5] Becker, R.; BraAck, M. A finite element pressure gradient stabilization for the Stokes equations based on local projections. Calcolo, 38(4):173-199, 2001.

[6] Bertoluzza, S. The discrete commutator property of approximation spaces. C. R. Acad. Sci. Paris Sér. I Math., 329(12):1097-1102, 1999. 
[7] Boris, J. P.; Grinstein, F. F.; Oran, E. S.; Kolbe, R. L. New insights into large eddy simulation. Fluid Dynamics Research, 10(4-6):199, 1992.

[8] Brenner, S. C.; Scott, L. R. The mathematical theory of finite element methods, volume 15 of Texts in Applied Mathematics. Springer, New York, third edition, 2008.

[9] Caffarelli, L.; Kohn, R.; Nirenberg, L. Partial regularity of suitable weak solutions of the Navier-Stokes equations. Comm. Pure Appl. Math., 35(6):771-831, 1982.

[10] Codina, R.; Blasco, J. Stabilized finite element method for the transient Navier-Stokes equations based on a pressure gradient projection. Computer Methods in Applied Mechanics and Engineering, 182:277-300, 2000.

[11] Codina, R.; Principe, J.; Guasch, O. Badia, S. Time dependent subscales in the stabilized finite element approximation of incompressible flow problems. Computer Methods in Applied Mechanics and Engineering, 196(2124):2413-2430, 2007.

[12] Colomés, O; Badia, S.; Codina, R.; Principe, J. Assessment of variational multiscale models for the large eddy simulation of turbulent incompressible flows. Computer Methods in Applied Mechanics and Engineering, 285:32-63, March 2015.

[13] Dauge, M. Stationary Stokes and Navier-Stokes systems on two- or three-dimensional domains with corners. I. Linearized equations. SIAM J. Math. Anal., 20(1):74-97, 1989.

[14] Ern, A.; And Guermond, J.-L. Theory and practice of finite elements, volume 159 of Applied Mathematical Sciences. Springer-Verlag, New York, 2004.

[15] Girault, V.; Raviart, P.-A. Finite element approximation of the Navier-Stokes equations, volume 749 of Lecture Notes in Mathematics. Springer-Verlag, Berlin-New York, 1979.

[16] Girault, V.; Raviart, P.-A. Finite element methods for Navier-Stokes equations, volume 5 of Springer Series in Computational Mathematics. Springer-Verlag, Berlin, 1986. Theory and algorithms.

[17] Grinstein, F. F.; Margoldin, L. G.; Rider, W. J. Implicit large eddy simulation: computing turbulent fluid dynamics. Cambridge university press, 2007.

[18] Grisvard, P. Elliptic problems in nonsmooth domains, volume 24 of Monographs and Studies in Mathematics. Pitman (Advanced Publishing Program), Boston, MA, 1985.

[19] Guasch, O.; Codina, R. Statistical behavior of the orthogonal subgrid scale stabilization terms in the finite element large eddy simulation of turbulent flows. Computer Methods in Applied Mechanics and Engineering, 261-262:154166, July 2013.

[20] Guermond, J.-L. Stabilization of Galerkin approximations of transport equations by subgrid modeling. ESAIM: Mathematical Modelling and Numerical Analysis - Modélisation Mathématique et Analyse Numérique, 33(6):1293$1316,1999$.

[21] Guermond, J.-L. Finite-element-based Faedo-Galerkin weak solutions to the Navier-Stokes equations in the threedimensional torus are suitable. Journal de Mathématiques Pures et Appliquées, 85(3):451-464, March 2006.

[22] Guermond, J.-L. Faedo-Galerkin weak solutions of the Navier-Stokes equations with Dirichlet boundary conditions are suitable. J. Math. Pures Appl. (9), 88(1):87-106, 2007.

[23] Guermond, J.-L. On the use of the notion of suitable weak solutions in CFD. International Journal for Numerical Methods in Fluids, 57(9):1153-1170, July 2008.

[24] Guermond, J.-L. The LBB condition in fractional Sobolev spaces and applications. IMA J. Numer. Anal., 29(3):790-805, 2009.

[25] Guermond, J.-L.; Pasciak, J. E. Stability of discrete Stokes operators in fractional Sobolev spaces. J. Math. Fluid Mech., 10(4):588-610, 2008.

[26] Hopf, E. Über die Anfangswertaufgabe für die hydrodynamischen Grundgleichungen. Math. Nachr., 4:213-231, 1951.

[27] Hughes, T. J. R.; Feijóo, G. R.; Mazzei, L.; Quincy, J.-B. The variational multiscale method - A paradigm for computational mechanics. Computer Methods in Applied Mechanics and Engineering, 166(1-2):3-24, 1998.

[28] Hughes, T. J. R.; Mazzei, L.; Jansen, K. E. Large eddy simulation and the variational multiscale method. Computing and Visualization in Science, 3:47-59, 2000.

[29] KellogG, R. B.; Osborn, J. E. A regularity result for the Stokes problem in a convex polygon. J. Functional Analysis, 21(4):397-431, 1976.

[30] Leray, J. Sur le mouvement d'un liquide visqueux emplissant l'espace. Acta Math., 63(1):193-248, 1934.

[31] Lin, F. H. A new proof of the Caffarelli-Kohn-Nirenberg theorem. Comm. Pure Appl. Math., 51(3):241-257, 1998.

[32] Lions, J.-L. Quelques méthodes de résolution des problèmes aux limites non linéaires. Dunod; Gauthier-Villars, Paris, 1969

[33] Lions, J.-L.; Magenes, E. Problèmes aux limites non homogènes et applications. Vol. 1. Travaux et Recherches Mathématiques, No. 17. Dunod, Paris, 1968.

[34] Scheffer, V. Hausdorff measure and the Navier-Stokes equations. Comm. Math. Phys., 55(2):97-112, 1977.

[35] Simon, J. Nonhomogeneous viscous incompressible fluids: existence of velocity, density, and pressure. SIAM J. Math. Anal., 21(5):1093-1117, 1990.

[36] Temam, R. Navier-Stokes equations. AMS Chelsea Publishing, Providence, RI, 2001. Theory and numerical analysis, Reprint of the 1984 edition. 